\title{
Modeling and Parameter Estimation of Hurricane Wind Fields with Asymmetry
}

\author{
DEREK CHANG \\ Center for Computational Engineering, Massachusetts Institute of Technology, Cambridge, Massachusetts \\ SAURABH AMIN \\ Department of Civil and Environmental Engineering and Institute for Data, Systems, and Society, Massachusetts \\ Institute of Technology, Cambridge, Massachusetts \\ KERRY EMANUEL \\ Program in Atmospheres, Oceans, and Climate, Massachusetts Institute of Technology, Cambridge, Massachusetts
}

(Manuscript received 29 May 2019, in final form 14 February 2020)

\begin{abstract}
This article presents an azimuthally asymmetric gradient hurricane wind field model that can be coupled with hurricane-track models for engineering wind risk assessments. The model incorporates low-wavenumber asymmetries into the maximum wind intensity parameter of the Holland et al. wind field model. The amplitudes and phases of the asymmetries are parametric functions of the storm-translation speed and wind shear. Model parameters are estimated by solving a constrained, nonlinear least squares (CNLS) problem that minimizes the sum of squared residuals between wind field intensities of historical storms and modelestimated winds. There are statistically significant wavenumber-1 asymmetries in the wind field resulting from both storm translation and wind shear. Adding the translation vector to the wind field model with wavenumber-1 asymmetries further improves the model's estimation performance. In addition, inclusion of the wavenumber- 1 asymmetry resulting from translation results in a greater decrease in modeling error than does inclusion of the wavenumber-1 shear-induced asymmetry. Overall, the CNLS estimation method can handle the inherently nonlinear wind field model in a flexible manner; thus, it is well suited to capture the radial variability in the hurricane wind field's asymmetry. The article concludes with brief remarks on how the CNLS-estimated model can be applied for simulating wind fields in a statistically generated ensemble.
\end{abstract}

\section{Introduction}

Hurricanes are a major natural hazard to built infrastructure such as buildings, transportation systems, and electric power networks (Campbell and Lowry 2012; Ouyang 2014) and often lead to large socioeconomic losses. A standardized risk assessment procedure is needed to assess the vulnerability of infrastructure systems to hurricanes. Such a procedure becomes especially important in light of projected changes in the frequency of high-intensity hurricanes (Bender et al. 2010). Hurricane risk assessment typically involves modeling of storm tracks, wind fields, and occurrence frequency (Watson and Johnson 2004). Outputs of these models are used toward quantifying the probability distributions of damage to various infrastructures.

Corresponding author: Derek Chang, changd@mit.edu
A common approach to risk assessment is to simulate an ensemble of storms, in order to capture the stochastic nature of hurricane arrival, landfall location, and intensity. This simulation-based approach relies on capturing two aspects of hurricane structure: 1) track, or trajectory of the storm from its formation over the ocean to dissipation over land; and 2) wind velocities at various points along the track, modeled by either a single intensity measurement (e.g., maximum sustained $10 \mathrm{~m}$ wind speed) or a surface wind field. Well-known synthetic-track-generation models combine both physical and statistical modeling approaches (Casson and Coles 2000; Vickery et al. 2000b; Emanuel et al. 2006) and use historical hurricane "best track" data as input. Maximum wind intensity estimates along the tracks are provided by models such as the Coupled Hurricane Intensity Prediction System (CHIPS) (Emanuel et al. 2004) or FAST intensity simulator (Emanuel 2017). 
Wind field models include those proposed by Vickery et al. (2000a), Emanuel (2004), Willoughby et al. (2006), Holland et al. (2010), and Chavas et al. (2015). For risk assessment of large-scale infrastructure systems, the impact of spatially heterogeneous wind velocities must be suitably captured. Thus, a reliable model of the whole surface wind field is desirable.

This article focuses on constructing a physically informed model of the hurricane gradient wind field; one can then use a boundary layer model for the gradient-tosurface wind conversion. Well-known gradient wind field models are estimated from deterministic axisymmetric models, which we refer to as mean field (MF) models. In reality, however, hurricane wind fields tend to be asymmetric. Not accounting for this inherent asymmetry can negatively affect hurricane risk assessment of aboveground infrastructure components such as electric distribution lines, whose failure probabilities depend on local surface wind intensities (Zhou et al. 2006; Alvehag and Soder 2011). The relationships of asymmetries to environmental inputs were studied in Xie et al. (2006), Uhlhorn et al. (2014), Vukicevic et al. (2014), and Klotz and Jiang (2017). This article extends on the previous work by modeling wind field asymmetries as a function of environmental inputs, specifically, by parametric incorporation of asymmetries into established models for mean field estimation.

In particular, our asymmetric wind field model is an extension of the mean field model of Holland et al. (2010) in that the model's maximum intensity parameter is set to be a harmonic function of the azimuthal angle. The asymmetries are quantified in terms of amplitudes and phases of the harmonic function to produce velocity estimates that are both radially and azimuthally varying. This asymmetry correction to the maximum intensity is general in that the amplitudes and phases are modeled as parametric functions of one or more environmental input(s). We use storm translation and environmental shear as inputs; see section 2 for a full model description.

The parameters that model the amplitudes and phases in the asymmetry correction are estimated by solving a constrained, nonlinear least squares (CNLS) problem. Solving this problem entails minimizing the sum of squared errors between velocity estimates and historical storm data for the region between the storm center and a predecided "cutoff" radius in the storm's outer region. The method, as discussed in section 3, can jointly estimate the amplitude and phase parameters while accounting for the nonlinearity of the asymmetric wind field model and parameter constraints. While we consider the Holland mean field model here due to its relative simplicity, the proposed CNLS formulation can be adapted to other mean field models.
The proposed approach captures the asymmetric wind field for the purpose of simulation, rather than for only hindcasting or nowcasting. Some previous works have also introduced asymmetries into an existing mean field model by considering that the mean field parameter(s) vary with azimuth. In particular, Xie et al. (2006) introduce a real-time nowcasting asymmetric model, but that model cannot be readily used to simulate a wind field ensemble. Uhlhorn et al. (2014) and Vukicevic et al. (2014) quantify asymmetries in storms using the semispectral approach, but their focus is on hindcasting and they do not explicitly parameterize asymmetries in wind field models.

In section 4, we present the setup for estimation and evaluation of the asymmetric wind field model, using multiple sources of hurricane wind data. In section 5, we discuss the performance of this model and highlight some observations about the relationship between asymmetries and environmental inputs. In section 6 , we briefly discuss how the model can be useful in wind field simulation along tracks in a statistically generated ensemble. Section 7 summarizes the results and proposes future directions of study.

\section{Hurricane wind field model}

\section{a. Mean field model}

Given a simulated track produced from a trackgeneration model, the Holland et al. (2010) model can be used to estimate the mean tangential wind at points along the track (see Fig. 1):

$$
V(r)=V_{m}\left\{\left(\frac{R_{m}}{r}\right)^{B} \exp \left[1-\left(\frac{R_{m}}{r}\right)^{B}\right]\right\}^{S(r)},
$$

where

$$
S(r)=\left\{\begin{array}{ll}
0.5, & r \leq R_{m} \\
0.5+\left(r-R_{m}\right) \frac{S_{n}-0.5}{r_{n}-R_{m}}, & r>R_{m}
\end{array} .\right.
$$

The model relates the gradient wind velocity $V\left(\mathrm{~m} \mathrm{~s}^{-1}\right)$ to the radial distance from the storm center $r(\mathrm{~km})$. Model parameters are maximum velocity $V_{m}$, radius of maximum wind $R_{m}$, shape parameter $B$ governing wind decay rate from the storm center, and scaling parameter $S$ to adjust the profile shape. The parameter $S$ is fixed to 0.5 at radius $r \leq R_{m}$ and varies linearly with $r$ for $r>R_{m}$. In the function for radius $r>R_{m}, S_{n}$ is taken to be the value of $S$ at which the estimated Holland velocity $V(r)$ is equal to a wind velocity observation in the storm periphery at radius $r_{n}$. 

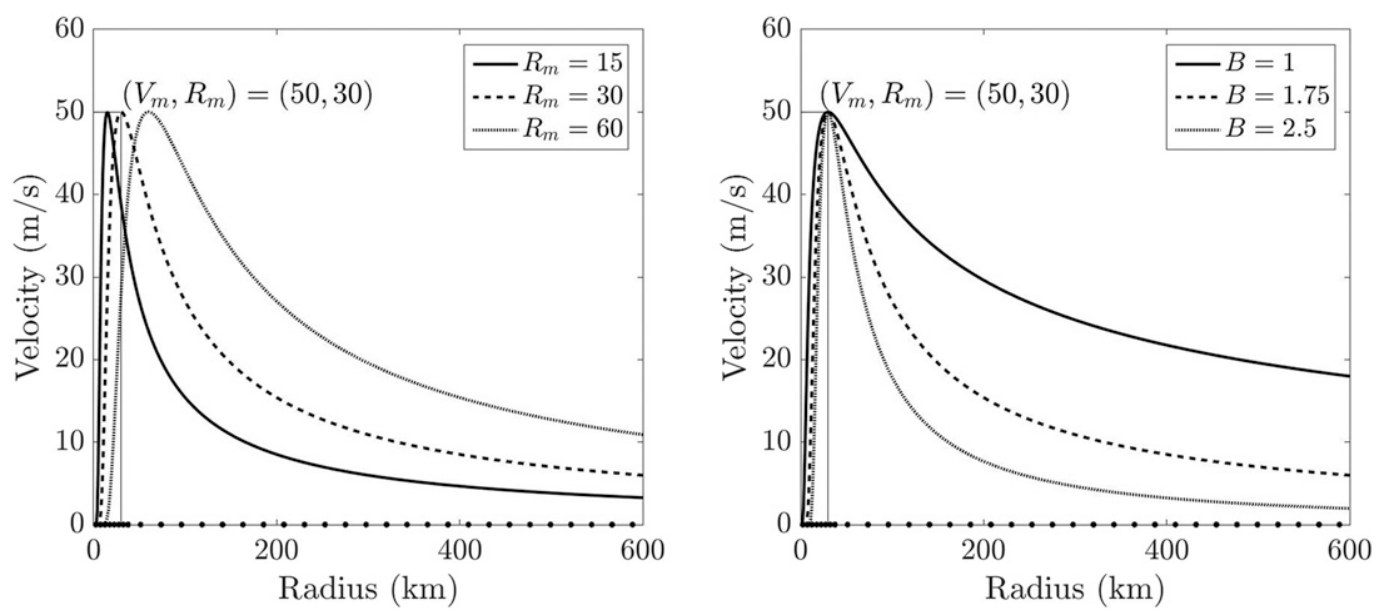

FIG. 1. Holland tangential velocity curves as a function of different (left) $R_{m}$ and (right) $B ;\left(V_{m}, R_{m}\right)=(50,30)$ for the dashed curve on the left plot and for all curves on the right plot, and the parameter $S$ is set to be $1 / 2$ at all values of radius $r$. The black dots on the $x$ axis mark the boundaries of the radius ranges used for binning in local averaging (section 4).

The Holland et al. (2010) model reduces to the original Holland (1980) model, when $S$ is fixed as 0.5 at all values of radius $r$. Willoughby and Rahn (2004) concluded that the original model tends to overestimate the wind's decay with radial distance; thus, it underestimates the wind velocities in the storm's outer regions. The revised 2010 model uses the radially varying scaling parameter $S$ in order to partially rectify these limitations.

For the purpose of modeling asymmetry in the wind field, we empirically estimate the mean field parameters for each snapshot. A snapshot is a depiction of the hurricane wind field at a point in time. We estimate the maximum wind intensity $V_{m}$ by averaging the velocity data points in the vicinity of the radius of maximum winds $R_{m}$ (i.e., $0.9 R_{m}-1.2 R_{m}$ ). Consequently, $V_{m}$ is effectively the azimuthally averaged maximum intensity. The parameter $R_{m}$ for each snapshot is taken to be the value of radius $r$ at which the maximum velocity in the snapshot occurs. Then, the remaining parameters $B$ and $S$ in Eq. (1) are chosen for each snapshot to best fit the data. In particular, we search over discrete values of $B$ in the range $0.5-4$; for each value of $B$, we take $r_{n}$ to be the maximum $r$ in the snapshot and obtain $S$ as a function of $r$ using Eq. (2). The $B$ and $S$ that minimize the absolute difference between the snapshot wind data and the Holland model estimates are chosen as the empirical model parameters for that snapshot. We note that while Holland (1980) suggests that $B$ be selected from the range of 1-2.5, we consider a wider range of $B$ to ensure that the estimated mean field best fits the data. Hereafter, the empirically estimated maximum intensity parameter for the mean field is referred to as $\bar{V}_{m}$, whereas $V_{m}$ refers to the maximum intensity as a function of azimuth, that is, the asymmetry model discussed in section $2 b$.

\section{b. Asymmetry model}

Before presenting our asymmetry model based on the Holland mean field model, we first summarize some of the previous approaches to model asymmetry in wind fields.

Xie et al. (2006) account for the asymmetry by setting $R_{m}$ in the Holland model to be a polynomial function of the azimuthal angle. This approach is shown to produce more accurate real-time forecasts than mean field models. Estimation of the function parameters requires forecasted values of storm wind velocities [radial extent of 34-, 50-, and 64-kt winds $\left(1 \mathrm{kt} \approx 0.51 \mathrm{~m} \mathrm{~s}^{-1}\right)$ ], which can be obtained from the National Hurricane Center tropical cyclone forecasts. However, such detailed information on wind velocities is not available as input in simulation applications; thus the approach by Xie et al. (2006) is not applicable for simulation of a representative wind field ensemble. A more closely related work is by Uhlhorn et al. (2014, hereinafter U14) who model asymmetry by representing $V_{m}$ as an azimuthally varying harmonic function. Specifically, in U14, $V_{m}$ is parameterized by the wavenumber- 0 mean field maximum velocity, and the wavenumber- 1 asymmetric amplitude and phase. Then, the linear relationships between the wavenumber-1 parameters (amplitude and phase) and environmental inputs (storm-translation speed and wind shear) at $R_{m}$ are estimated. It is well recognized that the main environmental inputs contributing to asymmetry are storm translation and wind shear (Shapiro 1983). 
We represent $V_{m}$ as a harmonic function in which the amplitude(s) and phase(s) are parameterized by storm translation $V_{\text {tr }}$ and shear $V_{\text {sh }}$, which are available, timedependent inputs. Hereinafter, we add subscripts tr for translation and sh for wind shear to azimuth $\lambda$, amplitude $A$, and phase $\phi$. Specifically, $\lambda_{\text {tr }}$ and $\lambda_{\text {sh }}$ are the azimuth defined clockwise from the translation and shear directions, respectively, whereas $A_{\mathrm{tr}}$ and $\phi_{\mathrm{tr}}$ define the asymmetry induced by translation and $A_{\mathrm{sh}}$ and $\phi_{\mathrm{sh}}$ define the asymmetry induced by shear. We model the maximum intensity $V_{m}$ as the following harmonic function:

$$
\begin{gathered}
V_{m}\left(\lambda_{\mathrm{tr}}, \lambda_{\mathrm{sh}} ; \bar{V}_{m}, V_{\mathrm{tr}}, V_{\mathrm{sh}}\right)=\bar{V}_{m}+A_{\mathrm{tr}}\left(V_{\mathrm{tr}}\right) \cos \left[\lambda_{\mathrm{tr}}-\right. \\
\left.\phi_{\mathrm{tr}}\left(V_{\mathrm{tr}}\right)\right]+A_{\mathrm{sh}}\left(V_{\mathrm{sh}}\right) \cos \left[\lambda_{\mathrm{sh}}-\phi_{\mathrm{sh}}\left(V_{\mathrm{sh}}\right)\right],
\end{gathered}
$$

to express the maximum intensity as a function of the variables (azimuths $\lambda_{\mathrm{tr}}$ and $\lambda_{\mathrm{sh}}$ ) and model inputs $\left(\bar{V}_{m}, V_{\mathrm{tr}}\right.$, and $\left.V_{\mathrm{sh}}\right)$. The parameter $\bar{V}_{m}$, which is the estimated maximum intensity in the mean field model, is the wavenumber- 0 component in the formulation.

In Eq. (3), the amplitudes and phases of the asymmetries are modeled as affine functions of translation and shear:

$$
\begin{aligned}
& A_{\mathrm{tr}}\left(V_{\mathrm{tr}}\right)=x_{\mathrm{tr}, A}^{(0)}+x_{\mathrm{tr}, A}^{(1)} V_{\mathrm{tr}}, \\
& \phi_{\mathrm{tr}}\left(V_{\mathrm{tr}}\right)=x_{\mathrm{tr}, \phi}^{(0)}+x_{\mathrm{tr}, \phi}^{(1)} V_{\mathrm{tr}}, \\
& A_{\mathrm{sh}}\left(V_{\mathrm{sh}}\right)=x_{\mathrm{sh}, A}^{(0)}+x_{\mathrm{sh}, A}^{(1)} V_{\mathrm{sh}}, \quad \text { and } \\
& \phi_{\mathrm{sh}}\left(V_{\mathrm{sh}}\right)=x_{\mathrm{sh}, \phi}^{(0)}+x_{\mathrm{sh}, \phi}^{(1)} V_{\mathrm{sh}} .
\end{aligned}
$$

The values of the estimated amplitudes are constrained to be strictly nonnegative:

$$
A_{\mathrm{tr}}\left(V_{\mathrm{tr}}\right) \geq 0 ; \quad A_{\mathrm{sh}}\left(V_{\mathrm{sh}}\right) \geq 0,
$$

where $A_{\mathrm{tr}}\left(V_{\mathrm{tr}}\right)$ is given by Eq. (4a) and $A_{\mathrm{sh}}\left(V_{\mathrm{sh}}\right)$ is given by Eq. (4c). Furthermore, the values of the estimated phases are constrained to be within a range that spans $2 \pi$ :

$b_{\phi, \mathrm{tr}} \leq \phi_{\mathrm{tr}}\left(V_{\mathrm{tr}}\right) \leq b_{\phi, \mathrm{tr}}+2 \pi ; \quad b_{\phi, \mathrm{sh}} \leq \phi_{\mathrm{sh}}\left(V_{\mathrm{sh}}\right) \leq b_{\phi, \mathrm{sh}}+2 \pi$,

where $\phi_{\mathrm{tr}}\left(V_{\mathrm{tr}}\right)$ is given by Eq. (4b) and $\phi_{\mathrm{sh}}\left(V_{\mathrm{sh}}\right)$ is given by Eq. (4d). The parameters $b_{\phi, \text { tr }}$ and $b_{\phi, \text { sh }}$ are the lower bounds of the ranges for translation and shear, respectively; thus, the respective ranges for the phases are $\left[b_{\phi, \mathrm{tr}}, b_{\phi, \mathrm{tr}}+2 \pi\right]$ and $\left[b_{\phi, \mathrm{sh}}, b_{\phi, \mathrm{sh}}+2 \pi\right]$.

In summary, the complete wind field model is defined by the mean field model [Eqs. (1) and (2)], the asymmetry model [Eqs. (3) and (4)], and the constraints [Eqs. (5) and (6)]. The asymmetry model involves a total of eight unknowns (parameters) - four to capture translation effects and four to capture shear effects.

Another way to account for the effect of translation would be to add the storm-translation vector to the wind field (Schwerdt et al. 1979; Vickery et al. 2009). The maximum velocity under equal radius when the stormtranslation vector is added to the mean field occurs at exactly $90^{\circ}$ clockwise of the translation direction, where the storm motion and cyclostrophic wind direction are aligned. However, U14 found that if the translationinduced asymmetry is modeled as a wavenumber-1 component, the azimuth corresponding to the maximum velocity (i.e., phase $\phi_{\text {tr }}$ ) varies linearly with translation speed. To ensure that the model-estimated and observationally based asymmetries are aligned, we model the asymmetry resulting from translation as a wavenumber-1 component with tunable parameters $\mathbf{x}_{\mathrm{tr}}$. In section $2 \mathrm{c}$, we discuss how our asymmetry model is extended to include the translation vector in addition to wavenumber- 1 asymmetries.

\section{c. Combined wind field model}

To present the combined mean field and asymmetry model, we denote the eight unknowns (parameters) in section $2 \mathrm{~b}$ as $\mathbf{x}_{\mathrm{tr}}=\left[x_{\mathrm{tr}, A}^{(0)}, x_{\mathrm{tr}, A}^{(1)}, x_{\mathrm{tr}, \phi}^{(0)}, x_{\mathrm{tr}, \phi}^{(1)}\right]$ and $\mathbf{x}_{\mathrm{sh}}=$ $\left[x_{\mathrm{sh}, A}^{(0)}, x_{\mathrm{sh}, A}^{(1)}, x_{\mathrm{sh}, \phi}^{(0)}, x_{\mathrm{sh}, \phi}^{(1)}\right]$. The full vector of unknowns is denoted as $\mathbf{x}=\left(\mathbf{x}_{\mathrm{tr}}, \mathbf{x}_{\mathrm{sh}}\right)$.

We can rewrite the combined wind field model as a function of the unknowns $\mathbf{x}$, variables (radial distance $r$ as well as azimuthal angles $\lambda_{\text {tr }}$ and $\lambda_{\mathrm{sh}}$ ), and input parameters to the mean field and asymmetry models. In the mean field model, velocity $V$ was expressed as a function of the radius $r$ [Eqs. (1) and (2)] and parameterized by empirically estimated Holland parameters $\bar{V}_{m}, R_{m}, B$, and $S(r)$. Furthermore, in the asymmetry model, the maximum intensity parameter $V_{m}$ in Eq. (1) was expressed as a function of azimuths $\lambda_{\mathrm{tr}}$ and $\lambda_{\mathrm{sh}}$, and parameterized by translation $V_{\mathrm{tr}}$, wind shear $V_{\mathrm{sh}}$, and the input parameter $\bar{V}_{m}$ [Eqs. (3) and (4)]. To summarize, the mean field is affected by the radius $r$ and Holland parameters $\mathbf{s}(r)=\left[R_{m}, B, S(r)\right]$, and the asymmetry model is affected by the azimuthal angles $\boldsymbol{\lambda}=\left(\lambda_{\mathrm{tr}}, \lambda_{\mathrm{sh}}\right)$, Holland parameter $\bar{V}_{m}$, and environmental inputs $\mathbf{u}=\left(V_{\mathrm{tr}}, V_{\mathrm{sh}}\right)$.

With a slight abuse of notation, we use $V[r, \boldsymbol{\lambda} ; \mathbf{s}(r)$, $\left.\bar{V}_{m}, \mathbf{u} ; \mathbf{x}\right]$ to refer to the velocity function. We use this notation to emphasize that the velocity is a function of variables $r$ and $\boldsymbol{\lambda}$; model inputs $\mathbf{s}(r), \bar{V}_{m}$, and $\mathbf{u}$; and unknown parameters $\mathbf{x}$. Then, we can write

$$
V\left[r, \boldsymbol{\lambda} ; \mathbf{s}(r), \bar{V}_{m}, \mathbf{u} ; x\right]=V_{m}\left(\lambda ; \bar{V}_{m}, \mathbf{u} ; \mathbf{x}\right) Y[r ; \mathbf{s}(r)],
$$

where 

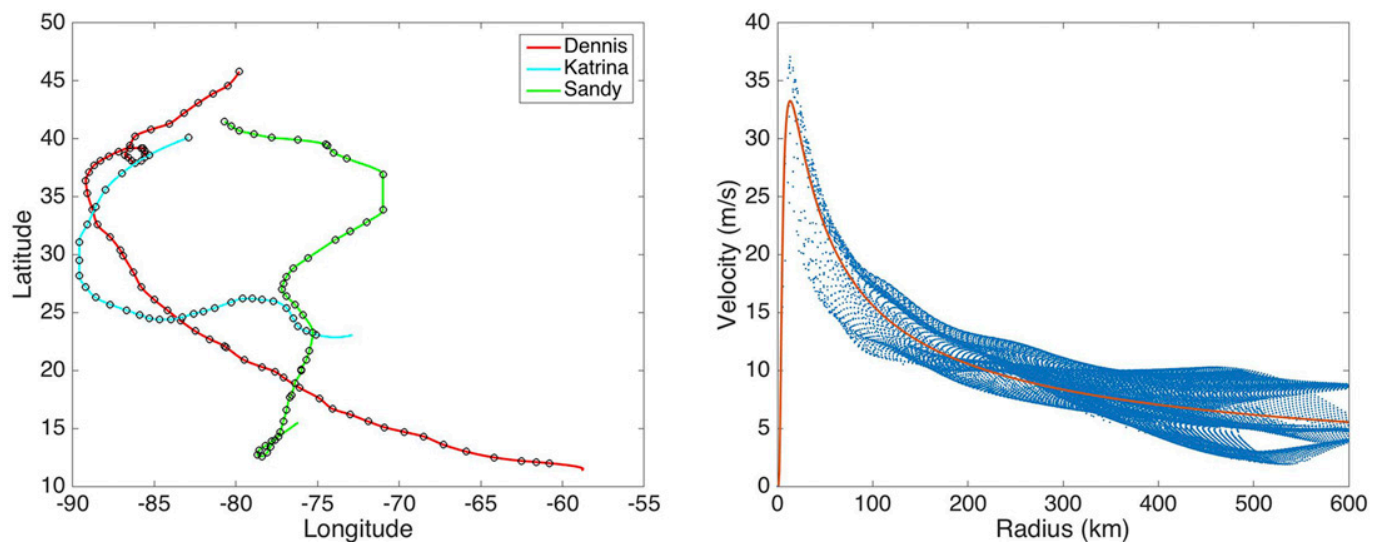

FIG. 2. (left) Examples of hurricane tracks, interpolated using HURDAT data. (right) An example of HWind velocity data (blue) from one snapshot and a best-fit Holland mean field estimate (red) as a function of radius.

$$
\begin{aligned}
V_{m}\left(\boldsymbol{\lambda} ; \bar{V}_{m}, \mathbf{u} ; \mathbf{x}\right)= & \bar{V}_{m}+A_{\mathrm{tr}}\left(V_{\mathrm{tr}} ; \mathbf{x}_{\mathrm{tr}, A}\right) \cos \left[\lambda_{\mathrm{tr}}-\right. \\
& \left.\phi_{\mathrm{tr}}\left(V_{\mathrm{tr}} ; \mathbf{x}_{\mathrm{tr}, \phi}\right)\right]+A_{\mathrm{sh}}\left(V_{\mathrm{sh}} ; \mathbf{x}_{\mathrm{sh}, A}\right) \cos \left[\lambda_{\mathrm{sh}}-\right. \\
& \left.\phi_{\mathrm{sh}}\left(V_{\mathrm{sh}} ; \mathbf{x}_{\mathrm{sh}, \phi}\right)\right] \quad \text { and } \\
Y[r ; \mathbf{s}(r)]= & \left\{\left(\frac{R_{m}}{r}\right)^{B} \exp \left[1-\left(\frac{R_{m}}{r}\right)^{B}\right]\right\}^{S(r)}
\end{aligned}
$$

Here, $\mathbf{x}_{\mathrm{tr}, A}=\left[x_{\mathrm{tr}, A}^{(0)}, x_{\mathrm{tr}, A}^{(1)}\right], \mathbf{x}_{\mathrm{tr}, \phi}=\left[x_{\mathrm{tr}, \phi}^{(0)}, x_{\mathrm{tr}, \phi}^{(1)}\right], \mathbf{x}_{\mathrm{sh}, A}=\left[x_{\mathrm{sh}, A}^{(0)}\right.$, $\left.x_{\mathrm{sh}, A}^{(1)}\right]$, and $\mathbf{x}_{\mathrm{sh}, \phi}=\left[x_{\mathrm{sh}, \phi}^{(0)}, x_{\mathrm{sh}, \phi}^{(1)}\right]$. Equation (7) can be rewritten as follows, to express the wind field model as the sum of the mean field winds and the asymmetries resulting from translation and shear:

$$
\begin{aligned}
V\left[r, \boldsymbol{\lambda} ; \mathbf{s}(r), \bar{V}_{m}, \mathbf{u} ; \mathbf{x}\right]= & V_{\mathrm{MF}}+V_{m, \mathrm{tr}}\left(\lambda_{\mathrm{tr}} ; V_{\mathrm{tr}} ; \mathbf{x}_{\mathrm{tr}}\right) Y[r ; \mathbf{s}(r)] \\
& +V_{m, \mathrm{sh}}\left(\lambda_{\mathrm{sh}} ; V_{\mathrm{sh}} ; \mathbf{x}_{\mathrm{sh}}\right) Y[r ; \mathbf{s}(r)],
\end{aligned}
$$

where

$$
\begin{aligned}
V_{\mathrm{MF}}= & \bar{V}_{m} Y[r ; \mathbf{s}(r)], \\
V_{m, \mathrm{tr}}\left(\lambda_{\mathrm{tr}} ; V_{\mathrm{tr}} ; \mathbf{x}_{\mathrm{tr}}\right)= & A_{\mathrm{tr}}\left(V_{\mathrm{tr}} ; \mathbf{x}_{\mathrm{tr}, A}\right) \cos \left[\lambda_{\mathrm{tr}}\right. \\
& \left.-\phi_{\mathrm{tr}}\left(V_{\mathrm{tr}} ; \mathbf{x}_{\mathrm{tr}, \phi}\right)\right], \quad \text { and } \\
V_{m, \mathrm{sh}}\left(\lambda_{\mathrm{sh}} ; V_{\mathrm{sh}} ; \mathbf{x}_{\mathrm{sh}}\right)= & A_{\mathrm{sh}}\left(V_{\mathrm{sh}} ; \mathbf{x}_{\mathrm{sh}, A}\right) \cos \left[\lambda_{\mathrm{sh}}\right. \\
& \left.-\phi_{\mathrm{sh}}\left(V_{\mathrm{sh}} ; \mathbf{x}_{\mathrm{sh}, \phi}\right)\right] .
\end{aligned}
$$

Here, $V_{\mathrm{MF}}$ denotes the mean field, $V_{m, \mathrm{tr}}\left(\lambda_{\mathrm{tr}} ; V_{\mathrm{tr}} ; \mathbf{x}_{\mathrm{tr}}\right)$ denotes the asymmetry resulting from translation, and $V_{m, \mathrm{sh}}\left(\lambda_{\mathrm{sh}} ; V_{\mathrm{sh}} ; \mathbf{x}_{\mathrm{sh}}\right)$ denotes the asymmetry resulting from shear. The estimated wind field velocities are 1-min sustained winds.
To obtain a simpler wind field model that considers only asymmetry resulting from the translation vector, one can just perform vector addition of the mean field and translation vector. The resultant asymmetric winds are referred to as $V_{\mathrm{MF}+\mathrm{TV}}{ }^{1}$ To obtain a model that considers both the translation vector and wavenumber-1 asymmetries, the terms $V_{m \text {,tr }}\left(\lambda_{\mathrm{tr}} ; V_{\mathrm{tr}} ; \mathbf{x}_{\mathrm{tr}}\right) Y[r ; \mathbf{s}(r)]$ and $V_{m, \mathrm{sh}}\left(\lambda_{\mathrm{sh}} ; V_{\mathrm{sh}} ; \mathbf{x}_{\mathrm{sh}}\right) Y[r ; \mathbf{s}(r)]$ are added to $V_{\mathrm{MF}+\mathrm{TV}}$. In section 5, we evaluate the effects of wavenumber-1 asymmetries and the translation vector on the model performance.

\section{d. Data}

We use data on hurricane tracks and wind field snapshots for parameter estimation (see Fig. 2 for plots of example tracks and snapshots). The National Hurricane Centers hurricane databases (HURDAT-2) is used for track locations of past storms (Landsea and Franklin 2013), which provide data every six hours on the storm's location and maximum sustained wind speed. The wind field data include 1) the Hurricane Research Division hurricane surface wind analysis system $\left[\mathrm{H}^{*}\right.$ Wind, now Risk Management Solutions, Inc., (RMS) HWind] radial structure database (Powell et al. 1998) and 2) output from the Pennsylvania State University Weather Research and Forecasting (WRF) Model-based ensemble Kalman filter (EnKF) data assimilation system (Weng and Zhang 2012). These datasets contain surface $(\sim 10 \mathrm{~m})$ wind velocity estimates obtained by processing direct observations through statistical models, data assimilation, and objective analyses. Although we use surface wind field data to estimate

\footnotetext{
${ }^{1}$ The velocities $V_{\mathrm{MF}+\mathrm{TV}}$ are dependent on the storm-translation magnitude $V_{\text {tr }}$ and direction $\theta_{\text {tr }}$, in addition to $\bar{V}_{m}, r, \mathbf{s}(r)$, and the azimuthal angle.
} 
parameters of a gradient wind field model, RousseauRizzi and Emanuel (2019) showed that the speed of surface winds is the same as that of the gradient wind in an axisymmetric, steady-state storm over the sea.

A subset of the wind field data is used for estimation of the asymmetric wind field model parameters, and the resulting model is evaluated by comparing to the remaining data. Only HWind snapshots were used for estimating model parameters. HWind snapshots are 1-min sustained winds, derived by assimilation and processing of in situ and remotely sensed observations. The snapshots are given at a typical interval of $3 \mathrm{~h}$ and horizontal grid spacing of $6 \mathrm{~km}$. Naturally, one would expect that the estimated parameters and asymmetry model are affected by the HWind data's inherent biases and variability. Total variability among observations at a particular radius ranges from $7 \%$ near the eyewall to about $15 \%$ in storm outer regions. There is a roughly $10 \%$ positive bias in wind speeds around the eyewall, and this bias decreases with $r$ (DiNapoli et al. 2012).

Both HWind and WRF data are used for evaluation of the estimated wind field model, mainly because WRF data are only available for two of the storms studied here (Katrina and Sandy) and thus not sufficient for parameter estimation. The WRF snapshots are instantaneous wind estimates produced by assimilating observations (e.g., airborne Doppler radar velocity data) into the convection-permitting WRF EnKF analysis. These snapshots are more fine-grained than HWind snapshots, occur at an interval of $30 \mathrm{~min}$ (Sandy) or $1 \mathrm{~h}$ (Katrina) and have horizontal grid spacing of around $3 \mathrm{~km}$ over an approximately $1700 \mathrm{~km} \times 1700 \mathrm{~km}$ region (Katrina) or $900 \mathrm{~km} \times 900 \mathrm{~km}$ region (Sandy). The intensity of simulated WRF storms is sensitive to the model's air-sea surface flux parameterization scheme (Green and Zhang 2013). In addition, WRF data for an ensemble of storms display large forecast track divergence due to uncertainties in environmental flow (Munsell and Zhang 2014). These sensitivities affect the comparative model performance of WRF and HWind data, which is reported in section $5 \mathrm{c}$. We converted the WRF data to 1-min sustained winds following Eq. (1) from Klotz and Nolan (2019).

\section{Parameter estimation}

This section details the CNLS optimization problem for parameter estimation of the asymmetric wind field model. The goal of a standard CNLS problem is to select unknown parameters $\mathbf{x}$ that minimize an objective function $f(\mathbf{x})$ while also satisfying predefined constraints that capture allowable bounds on the parameters. Specifically, $f(\mathbf{x})=1 / 2 \sum_{i=1}^{N} e_{i}^{2}(\mathbf{x})$ for $N$ data points, where $e_{i}(\mathbf{x})$ is the residual at data point $i$. Commonly used optimization solvers rely on the gradient and Hessian of the objective function to iteratively obtain estimates of the parameters until the objective function is minimized or the number of iterations is exceeded. The objective, gradient, and Hessian for a CNLS problem with unknown vector $\mathbf{x}$ of length $M$ are expressed as

$$
\begin{aligned}
f(\mathbf{x}) & =\frac{1}{2} \sum_{i=1}^{N} e_{i}^{2}(\mathbf{x}) \\
\nabla f(\mathbf{x}) & =J(\mathbf{x})^{\mathrm{T}} e(\mathbf{x}) \\
\nabla^{2} f(\mathbf{x}) & =J(\mathbf{x})^{\mathrm{T}} J(\mathbf{x})+\sum_{i=1}^{N} e_{i}(\mathbf{x}) \nabla^{2} e_{i}(\mathbf{x}),
\end{aligned}
$$

where the $N \times M$ matrix $J(\mathbf{x})$ is the Jacobian whose terms are derivatives of the residuals with respect to $\mathbf{x}$. In general, one can express the equality and inequality constraints as follows:

$$
\begin{aligned}
& g_{k}(\mathbf{x})=c_{k}, \quad k=\{1, \ldots, K\}, \\
& h_{l}(\mathbf{x}) \geq d_{l}, \quad l=\{1, \ldots, L\},
\end{aligned}
$$

where $g_{k}()$ and $h_{l}()$ are linear functions of $\mathbf{x}$ and $c_{k}$ and $d_{l}$ are constants.

We now specify the objective of the CNLS problem for the purpose of estimating the wind field model introduced in section 2. Recall that the unknowns in CNLS are the eight parameters in Eqs. (3) and (4). The objective is to minimize the sum of squared residuals. For the $i$ th data point, the residual between the observed velocity $V_{i}$ and model-estimated velocity $\hat{V}_{i}$ is $e_{i}(\mathbf{x})=V_{i}-\hat{V}_{i}$, in which $\hat{V}_{i}$ is assigned the value of the velocity function [Eq. (7) or Eq. (9)] evaluated for the $i$ th data point. Note that we replace $V_{\mathrm{MF}}$ with $V_{\mathrm{MF}+\mathrm{TV}}$ if the translation vector is included in the model. Henceforth, $t$ denotes a snapshot, and $t(i)$ is the snapshot index corresponding to data point $i$. Consider that there are $T$ snapshots and $N_{t}$ velocity measurements per snapshot $t$. Thus, the total number of data points $N$ is given by $\sum_{t} N_{t}$. The CNLS problem for estimating the optimal parameter vector is written as

$$
\begin{aligned}
\underset{\mathbf{x}}{\operatorname{Minimize}} f(\mathbf{x})= & \frac{1}{2} \sum_{i=1}^{N} e_{i}^{2}(\mathbf{x})=\frac{1}{2} \sum_{i=1}^{N}\left\{V_{i}-\hat{V}_{i}\left[r_{i}, \boldsymbol{\lambda}_{i} ; \mathbf{s}_{i}\left(r_{i}\right),\right.\right. \\
& \left.\left.\bar{V}_{m, t(i)}, \mathbf{u}_{t(i)} ; \mathbf{x}\right]\right\}^{2}
\end{aligned}
$$

subject to the constraints given by Eqs. (5) and (6) for each snapshot $t$, which restrict the range of allowable values that the unknown parameters $\mathbf{x}$ may take. The indices of data points are dropped hereinafter for notational simplicity. 
Parameter estimates are obtained using the MATLAB interior-point constrained nonlinear solver, which takes an initial parameter vector $\mathbf{x}$ and iteratively updates it to minimize the objective. We use the following fmincon options: interior-point algorithm, user-supplied gradient and Hessian, and maximum number of iterations set to $100 .^{2}$ (See appendix A for the gradient and Hessian derivations that are supplied to the solver.)

\section{Experimental design}

This section covers data preparation, CNLS parameter estimation, and model evaluation.

\section{a. Preparation of data}

Storm track and wind field data are required for estimation of the wind field model (see section $2 \mathrm{~d}$ ). This study uses HURDAT tracks from Hurricanes Andrew (1992), Isabel (2003), Dennis (2005), Katrina (2005), Wilma (2005), Irene (2011), Isaac (2012), Sandy (2012), and Ingrid (2013). HWind data are obtained for all storms and WRF data for Katrina and Sandy to represent the wind field. The Sandy WRF data used here are 10 members of the 60-member ensemble forecast (Munsell and Zhang 2014). All 10 ensemble members had relatively accurate landfall locations (track error of $233.8 \mathrm{~km}$ averaged over a time window of $6 \mathrm{~h}$ before and after landing). The Katrina WRF data used are from a single ensemble member (Green and Zhang 2013), as other WRF simulations of Katrina were run on different air-sea flux parameterization schemes. In total, two Katrina "storms" (one HWind and one WRF) and 11 Sandy storms (one HWind and $10 \mathrm{WRF}$ ) are used in this study. As a proxy for excluding times at which storms have undergone extratropical transition, snapshots at a latitude greater than $35^{\circ} \mathrm{N}$ are not included in the dataset.

The wind shear for all snapshots is calculated using Global Forecast System (GFS) operational analyses, which are given at $\sim 1^{\circ}$ resolution. The first step in calculating the wind shear is to subtract an estimate of the hurricane vortex streamfunction (Galarneau and Davis 2013) from the GFS wind field, in order to isolate the environmental winds. Then, the mean environmental winds at heights of 850 and $250 \mathrm{hPa}$ are calculated by averaging over a 200-km radius around the storm center, and the 850-250-hPa-wind shear vector (magnitude $V_{\mathrm{sh}}$ and direction $\theta_{\mathrm{sh}}$ ) is obtained by vector subtraction of the two means. We use the GFS analyses because these data provide a more fine-grained estimation of the wind shear. However, for comparison purposes, we discuss model performance using wind shear from both the GFS analyses

\footnotetext{
${ }^{2}$ Further details about the interior-point algorithm can be found in Nocedal and Wright (2006).
}

and Statistical Hurricane Intensity Prediction Scheme (SHIPS) database (DeMaria et al. 2005) in section 5.

Next, we interpolate the HURDAT track positions to half-hour intervals using a piecewise cubic Hermite interpolating polynomial (PCHIP) (Chavas et al. 2015). The storm-translation vector (magnitude $V_{\text {tr }}$ and direction $\left.\theta_{\mathrm{tr}}\right)$ is calculated using the interpolated storm center positions. Then, the wind shear vector is interpolated to half-hour intervals using PCHIP. The interpolated $V_{\text {tr }}$ and $V_{\text {sh }}$ are used as inputs to the CNLS problem.

Then, velocity data are locally averaged, because there are more data points at larger values of radius $r$ in both HWind and WRF snapshots. To prepare for local averaging, the azimuths $\lambda_{\mathrm{tr}}$ and $\lambda_{\mathrm{sh}}$ are calculated for all data points as angles clockwise from $\theta_{\mathrm{tr}}$ and $\theta_{\mathrm{sh}}$, respectively. Then, velocity data from each snapshot are binned in $r-\lambda_{\text {tr }}$ space, and the velocity data in each bin are averaged (see Fig. 1 for details regarding bins). These locally averaged velocities $V(\sim 800-900$ per snapshot), as well as the radii $r$ and azimuths $\lambda_{\text {tr }}$ and $\lambda_{\text {sh }}$ corresponding to the bins, are used as inputs to CNLS. As a result of local averaging, the velocity inputs to CNLS are equally distributed across the entire range of $r$. The final step is to estimate the Holland parameters $\mathbf{s}(r)$ and $\bar{V}_{m}$.

A remaining issue to address is that the asymmetry varies radially, but the amplitudes and phases in Eq. (3) are not parameterized by radius $r$. It is difficult to model amplitudes and phases as a function of $r$ using simple parametric forms. Instead, we only use velocities corresponding to radii less than a cutoff radius $R_{\text {cut }}$ as input for parameter estimation and evaluation, removing the need to parameterize asymmetry as a function of $r$. The radius $R_{\text {cut }}$ is defined as $\max \left[\min \left(r_{15}, 300\right), \min \left(r_{0.75} \bar{V}_{m}, 300\right)\right]$ where $r_{15}$ denotes the radius at which the mean field velocity is $15 \mathrm{~m} \mathrm{~s}^{-1}$ and $r_{0.75} \bar{V}_{m}$ denotes the radius at which the mean field velocity is $0.75 \bar{V}_{m}$. The radius $r_{15}$ is a conservative practical cutoff for hurricane risk analysis of above-ground infrastructure components, such as overhead electricity lines and poles. ${ }^{3}$ However, for snapshots with low maximum intensity parameter $\bar{V}_{m}$, a cutoff of $r_{15}$ would omit most of the data points in the snapshot. Thus, we also ensure that radial regions of the storm with mean field velocity greater than $0.75 \bar{V}_{m}$ are included, regardless of whether or not $r_{0.75 \bar{V}_{m}}$ is greater than $r_{15}$. Last, $R_{\text {cut }}$ is less than $300 \mathrm{~km}$ to ensure that peripheral storm regions are not included.

\footnotetext{
${ }^{3}$ Specifically, according to a model estimated using historical storm data that include wind data from hurricanes, tropical depressions, and tropical storms (Zhang et al. 2014), electricity distribution lines are expected to fail at a frequency that increases quadratically with wind speed above $20 \mathrm{~m} \mathrm{~s}^{-1}$ and are much less likely to fail below $20 \mathrm{~m} \mathrm{~s}^{-1}$. Another cutoff value might be appropriate in other contexts, such as thunderstorms or storm surge.
} 
TABLE 1. List of six wind field models evaluated. The asymmetric models are classified as $V_{\mathrm{tr}}$ models (consider only translation) or $V_{\mathrm{tr}}+$ $V_{\text {sh }}$ models (consider both translation and shear). Furthermore, the model names containing the terms TV denote inclusion of the translation vector and those containing WVN-1 denote inclusion of the wavenumber-1 component(s) resulting from translation and/or shear. The mean field and $V_{\mathrm{tr}}(\mathrm{TV})$ models serve as benchmark models; all other models have unknown parameters to be estimated using the CNLS method.

\begin{tabular}{llc}
\hline \hline \multicolumn{1}{c}{ Model } & \multicolumn{1}{c}{ Translation effects } & Shear effects \\
\hline 1) Mean field & & - \\
$V_{\mathrm{tr}}$ models & & - \\
2) $V_{\mathrm{tr}}(\mathrm{TV})$ & Translation vector & - \\
3) $V_{\mathrm{tr}}(\mathrm{WVN}-1)$ & Wavenumber 1 & - \\
4) $V_{\mathrm{tr}}(\mathrm{TV}+\mathrm{WVN}-1)$ & Translation vector and wavenumber 1 & Wavenumber 1 \\
$V_{\mathrm{tr}}+V_{\mathrm{sh}}$ models & & Wavenumber 1 \\
5) $V_{\mathrm{tr}}(\mathrm{TV})+V_{\mathrm{sh}}(\mathrm{WVN}-1)$ & Translation vector & Translation vector and wavenumber 1 \\
6) $V_{\mathrm{tr}}(\mathrm{TV}+\mathrm{WVN}-1)+V_{\mathrm{sh}}(\mathrm{WVN}-1)$ & & \\
\end{tabular}

\section{b. Parameter estimation}

Four CNLS-estimated asymmetric models with wavenumber- 1 asymmetries are estimated. These asymmetric models are compared to the two benchmark models: mean field and mean field plus translation vector (see Table 1 for the complete list of six models considered).

Parameter initializations for solving the CNLS problem are obtained following a two-step approach. First, we empirically estimate the translation- and shearrelative wavenumber- 1 amplitude and phase at the radius of maximum winds for each snapshot. Then we simply use the unconstrained least squares method to estimate $\mathbf{x}$, in which the estimated amplitudes and phases are the inputs. The resulting value of $\mathbf{x}$ is taken as the initial parameter vector for the CNLS problem.

Snapshots from Hurricanes Andrew, Dennis, Ingrid, Isaac, Sandy, and Wilma (all HWind data) are used to train the models. In addition, a few storms are set aside as held-out test data for evaluation of the models: Irene (HWind), Isabel (HWind), Katrina (HWind, WRF), and Sandy (HWind, WRF). The training-test split of the data is made (Table 2) so that the selected training storms are heterogeneous in: geographical track coverage (including land coverage), time of occurrence (month and year), and maximum intensity. ${ }^{4}$

\section{c. Evaluation of model performance}

We report three performance metrics as a measure of model performance: cross-validation error (CVE), Bayesian information criterion (BIC), and mean-square error (MSE) on the test data.

\footnotetext{
${ }^{4}$ Note that HWind snapshots of Hurricane Sandy have been split across the training and test sets. The radial extent of Sandy (HWind) is unusually large at northerly latitudes relative to the other HWind storms studied here, so HWind snapshots of Sandy are split among training and testing data to account for this feature of storm structure in parameter estimation.
}

The cross-validation error is obtained from a $k$-fold cross-validation procedure. To perform $k$-fold cross validation, we partition the set of training snapshots into $k=5$ subsets (folds). Then, five sets of data are formed, in which each set consists of the training data with one fold of snapshots omitted. For each set of data, we obtain the MSE on the omitted fold using the model estimated with the entire training set. The cross-validation error of a model is equal to the MSE averaged over the five folds. This process of parameter estimation followed by evaluation on the held-out fold is conducted for each of the six models.

The Bayesian information criterion is a metric that rewards a model's goodness of fit, but also includes an

TABLE 2. Key characteristics of storms used for training and testing. The latitude range is the range covered by the snapshots and is not the actual range traveled by the storm. Maximum intensity metrics are obtained from HURDAT data and are given in knots. All storms use HWind, except for two test storms labeled as WRF.

\begin{tabular}{|c|c|c|c|c|}
\hline & $\begin{array}{c}\text { Max } \\
\text { intensity }\end{array}$ & $\begin{array}{c}\text { Lat } \\
\text { range }\left({ }^{\circ} \mathrm{N}\right)\end{array}$ & Time & $\begin{array}{c}\text { No. } \\
\text { snapshots }\end{array}$ \\
\hline \multicolumn{5}{|l|}{ Training set } \\
\hline Andrew & 150 & $25.38-25.64$ & Aug 1992 & 4 \\
\hline Dennis & 130 & $15.77-31.15$ & Jul 2005 & 28 \\
\hline Ingrid & 75 & $19.11-23.79$ & Sep 2013 & 12 \\
\hline Isaac & 70 & $14.70-29.91$ & $\begin{array}{c}\text { Aug-Sep } \\
2012\end{array}$ & 41 \\
\hline Sandy & 100 & $13.58-39.76$ & $\begin{array}{c}\text { Oct-Nov } \\
2012\end{array}$ & 21 \\
\hline Wilma & 160 & $15.68-28.51$ & Oct 2005 & 30 \\
\hline \multicolumn{5}{|l|}{ Test set } \\
\hline Irene & 105 & $15.08-41.80$ & Aug 2011 & 27 \\
\hline Isabel & 145 & $21.30-33.95$ & Sep 2003 & 27 \\
\hline Katrina & 175 & $24.30-25.99$ & Aug 2005 & 16 \\
\hline Katrina (WRF) & 175 & $24.40-38.34$ & Aug 2005 & 120 \\
\hline Sandy & 100 & $13.58-37.02$ & $\begin{array}{c}\text { Oct-Nov } \\
2012\end{array}$ & 20 \\
\hline Sandy (WRF) & 100 & $24.6-41.86$ & $\begin{array}{c}\text { Oct-Nov } \\
2012\end{array}$ & 256 \\
\hline
\end{tabular}


overfitting penalty that is an increasing function of the number of parameters (Burnham and Anderson 2004). One can assess the relative likelihoods of two models by using their BIC (see appendix B). Then, the models' MSEs evaluated on the held-out test data are intended to validate the expected model performance suggested by the CVE and BIC.

\section{Results}

This section discusses the CNLS-estimated parameters of the asymmetric wind field models, then compares the performance of the models. See Table 3 for the CNLS parameter estimates.

\section{a. Parameter estimates of $V_{\mathrm{tr}}$ models}

Here, we discuss the parameter estimates of two CNLSestimated models that incorporate asymmetry resulting from translation: the $V_{\mathrm{tr}}$ (WVN-1) model-asymmetry modeled as the wavenumber-1 (WVN-1) component; and the $V_{\text {tr }}(\mathrm{TV}+\mathrm{WVN}-1)$ model-asymmetry modeled as the translation vector (TV) and the wavenumber-1 component. We compare these models with the $V_{\text {tr }}(\mathrm{TV})$ benchmark model-asymmetry modeled as only the translation vector added to the mean field.

The parameters $\hat{\mathbf{x}}_{\mathrm{tr}}$ of the $V_{\mathrm{tr}}(\mathrm{WVN}-1)$ model demonstrate a statistically significant $(>95 \%$ confidence) linear relationship between translation speed $V_{\text {tr }}$ and both amplitude $A_{\mathrm{tr}}$ and phase $\phi_{\mathrm{tr}}$. The key difference between the $V_{\mathrm{tr}}(\mathrm{TV})$ model and the $V_{\mathrm{tr}}(\mathrm{WVN}-1)$ model is the azimuth corresponding to the maximum intensity. In the $V_{\text {tr }}(\mathrm{TV})$ model, the maximum velocity under equal radius occurs at $90^{\circ}$ clockwise of the translation direction, as was discussed in section 2. In the $V_{\mathrm{tr}}(\mathrm{WVN}-$ 1 model), the maximum velocity under equal radius occurs slightly clockwise of $90^{\circ}$ because of the hurricane inflow component.

The key difference between our result and U14's result is that we find a significant downwind storm-motionrelative phase rotation with increasing translation speed [i.e., the slope parameter $\hat{x}_{\mathrm{tr}, \phi}^{(1)}<0$ ]. In contrast, U14 find an upwind phase rotation $\left[\hat{x}_{\mathrm{tr}, \phi}^{(1)}>0\right]$. This discrepancy can be attributed to different parameter estimation methods. U14 use unconstrained least squares to estimate $\mathbf{x}_{\mathrm{tr}}$, in which the inputs are empirically estimated wavenumber- 1 amplitudes and phases at the radius of maximum winds. In contrast, the inputs for CNLS are observed velocities that lie within the cutoff radius $R_{\text {cut }}$. Indeed, we find a significant upwind storm-motion-relative phase rotation with translation speed $\left[\hat{x}_{\mathrm{tr}, \phi}^{(1)}=3.4^{\circ}\right]$ when applying U14's procedure.

The parameters of the $V_{\mathrm{tr}}(\mathrm{TV}+\mathrm{WVN}-1)$ model also demonstrate a significant relationship between translation

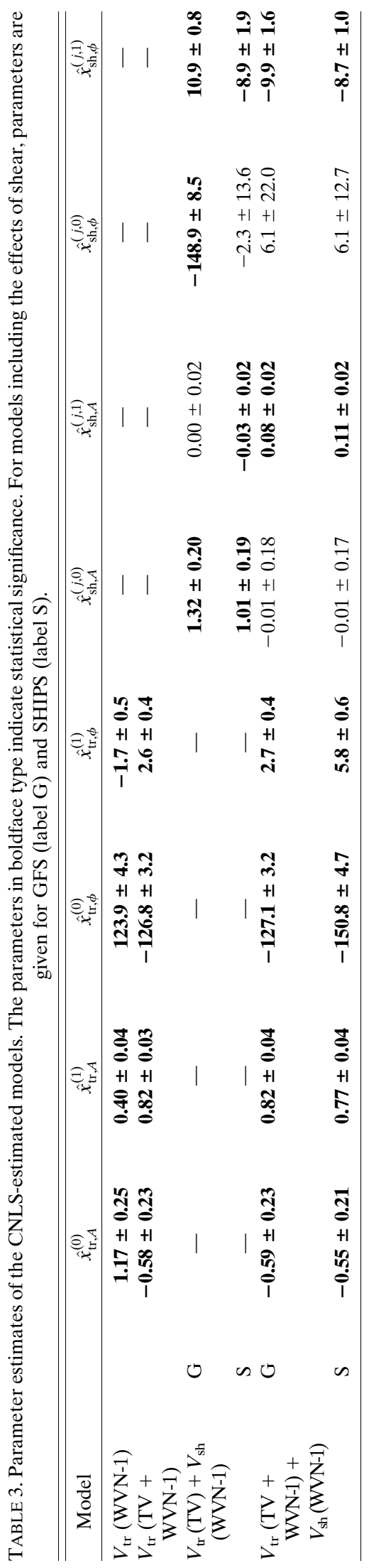


TABLE 4. Performance of models measured in terms of CVE and MSE on test storms. The CVE and MSE of models including asymmetry resulting from shear are given for GFS (label G) and SHIPS (label S). The best-performing model under each metric is in boldface type. MSE results for Sandy WRF are given as averaged MSE across 10 ensemble members.

\begin{tabular}{|c|c|c|c|c|c|c|c|c|}
\hline \multirow[b]{2}{*}{ Model } & & \multirow[b]{2}{*}{ CVE } & \multicolumn{4}{|c|}{ MSE (test storms) HWind } & \multicolumn{2}{|c|}{$\begin{array}{c}\text { MSE } \\
\text { (test storms) WRF }\end{array}$} \\
\hline & & & Irene & Isabel & Katrina & Sandy & Katrina & Sandy \\
\hline Mean field & & 33.2 & 33.6 & 32.9 & 8.9 & 31.4 & 64.7 & 65.4 \\
\hline$V_{\text {tr }}(\mathrm{WVN}-1)$ & & 29.5 & 24.0 & 29.4 & 10.8 & 28.7 & 74.3 & 54.2 \\
\hline$V_{\mathrm{tr}}(\mathrm{TV})$ & & 25.9 & 23.7 & 28.4 & 16.7 & 32.9 & 75.3 & 126.9 \\
\hline$V_{\mathrm{tr}}(\mathrm{TV}+\mathrm{WVN}-1)$ & & 20.0 & 25.7 & 26.2 & 11.6 & 25.3 & 72.1 & 77.7 \\
\hline \multirow[t]{2}{*}{$V_{\mathrm{tr}}(\mathrm{TV})+V_{\mathrm{sh}}(\mathrm{WVN}-1)$} & G & 25.7 & 23.6 & 28.0 & 18.5 & 33.2 & 77.4 & 124.0 \\
\hline & $\mathrm{S}$ & 21.4 & 15.7 & 28.9 & 17.1 & 23.6 & 95.2 & 92.2 \\
\hline \multirow[t]{2}{*}{$V_{\mathrm{tr}}(\mathrm{TV}+\mathrm{WVN}-1)+V_{\mathrm{sh}}(\mathrm{WVN}-1)$} & $\mathrm{G}$ & 19.9 & 26.2 & 26.1 & 11.6 & 24.9 & 71.0 & 77.4 \\
\hline & $\mathrm{S}$ & 17.2 & 16.7 & 25.9 & 11.4 & 16.8 & 95.2 & 90.7 \\
\hline
\end{tabular}

speed and both amplitude and phase. In this model, the wavenumber-1 parameters $\hat{\mathbf{x}}_{\text {tr }}$ estimate the residual asymmetry resulting from translation that remains after the translation vector has been added to the mean field. The phase associated with this residual asymmetry is more than $90^{\circ}$ counterclockwise from the translation direction $\left[\hat{x}_{\text {tr } \phi}^{(0)}=-126.8^{\circ}\right]$ and rotates upwind with increasing translation speed $\left[\hat{x}_{\mathrm{tr}, \phi}^{(1)}>0\right]$.

\section{b. Parameter estimates of $V_{\mathrm{tr}}+V_{\mathrm{sh}}$ models}

Two CNLS-estimated models with translation- and shear-induced asymmetries $\left(V_{\mathrm{tr}}+V_{\mathrm{sh}}\right.$ models $)$ are discussed here: $V_{\mathrm{tr}}(\mathrm{TV})+V_{\mathrm{sh}}(\mathrm{WVN}-1)$ model-asymmetry
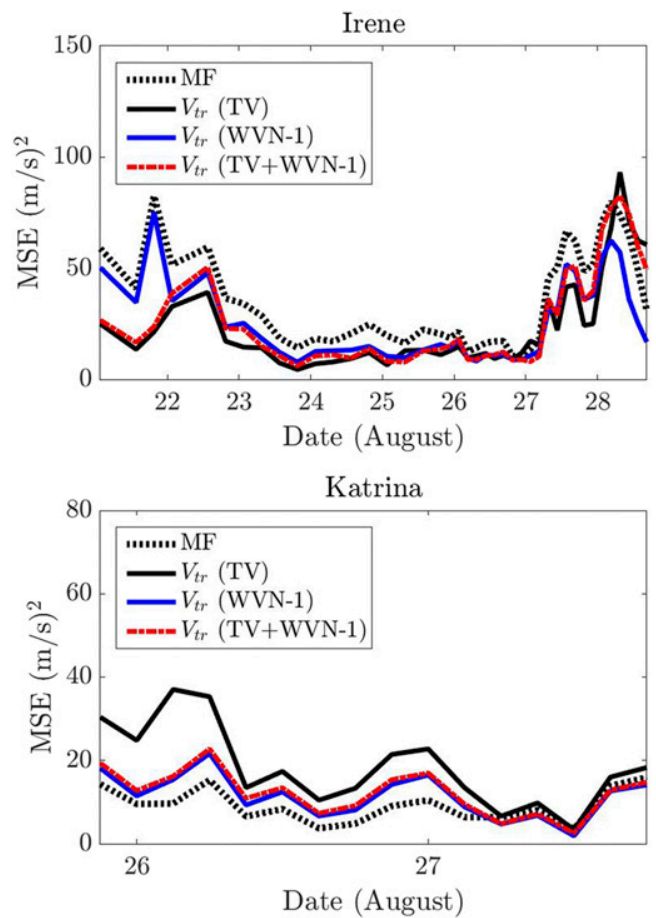

modeled as the translation vector (TV) and wavenumber-1 component resulting from shear; and $V_{\text {tr }}(\mathrm{TV}+\mathrm{WVN}-1)+$ $V_{\text {sh }}$ (WVN-1) model-asymmetry modeled as the translation vector and wavenumber-1 component resulting from both translation and shear. The parameters $\hat{\mathbf{x}}_{\mathrm{sh}}$ of both models demonstrate a significant linear relationship between shear magnitude and both amplitude $A_{\text {sh }}$ and phase $\phi_{\text {sh }}$.

The phase parameters $\hat{\mathbf{x}}_{\mathrm{sh}, \phi}$ estimated using SHIPS data demonstrate that the phase rotates downwind from downshear to left of shear with increasing shear magnitude $\left[\hat{x}_{\mathrm{sh}, \phi}^{(1)}<0\right.$ for both models]. This result is in agreement with that of U14, who also used SHIPS data.
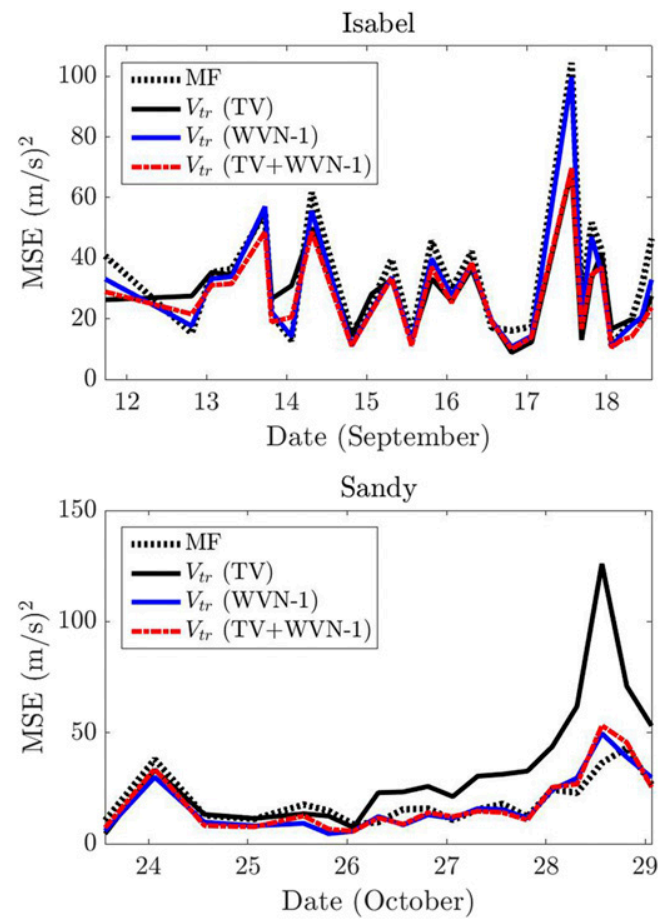

FIG. 3. Performance of the mean field and $V_{\mathrm{tr}}$ models on HWind testing storms, in terms of MSE. 

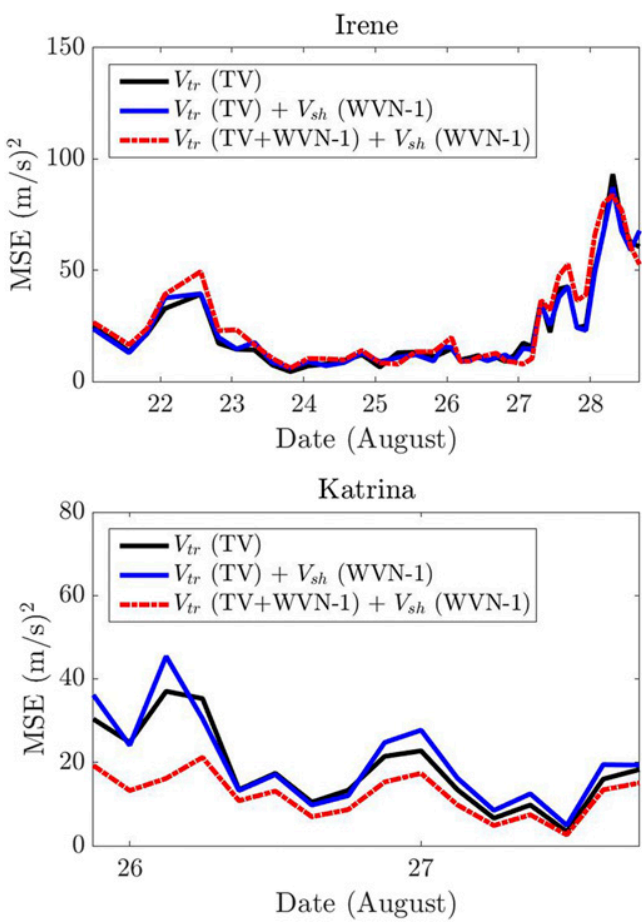
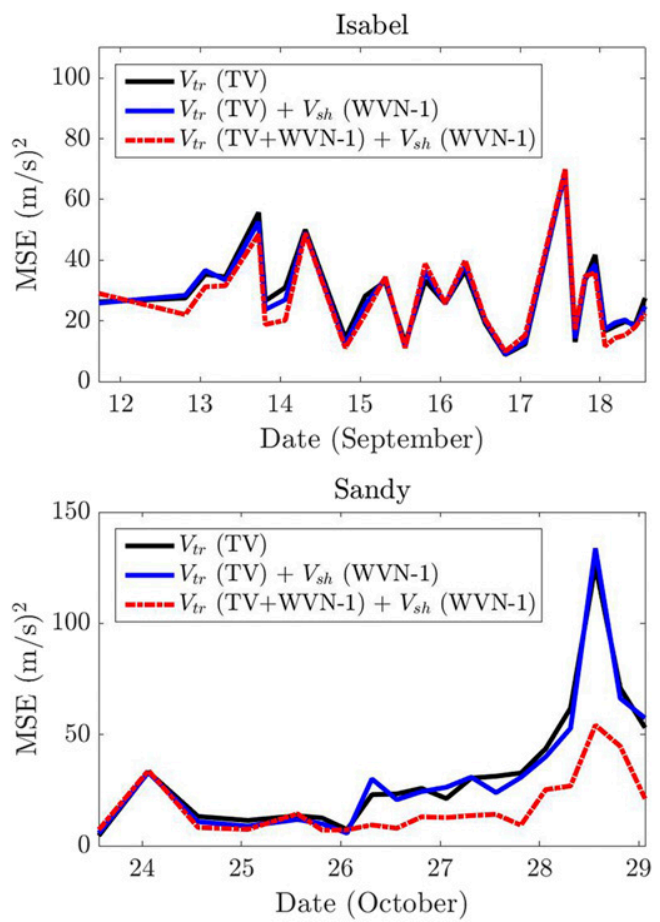

FIG. 4. Performance of the $V_{\mathrm{tr}}(\mathrm{TV})$ model and $V_{\mathrm{tr}}+V_{\mathrm{sh}}$ models on HWind testing storms and using GFS data for shear, in terms of MSE.

However, in contrast to our approach, U14 subtract only the estimated wavenumber-1 asymmetry resulting from translation from the storm winds, before estimating the wavenumber-1 asymmetry resulting from shear. They did not consider the translation vector because the wind data they used did not provide the wind direction.

The parameters of the $V_{\mathrm{tr}}(\mathrm{TV}+\mathrm{WVN}-1)+V_{\mathrm{sh}}$ (WVN-1) model using GFS also indicate a downwind rotation of the phase from downshear to left of shear. However, the parameters of the $V_{\mathrm{tr}}(\mathrm{TV})+V_{\mathrm{sh}}(\mathrm{WVN}-1)$ model instead indicate that the phase rotates upwind $\left[\hat{x}_{\mathrm{sh}, \phi}^{(1)}>0\right]$, from upshear left $\left[-180^{\circ}<\phi_{\mathrm{sh}}^{(j)}<-90^{\circ}\right]$ to downshear left $\left[-90^{\circ}<\phi_{\mathrm{sh}}^{(j)}<0^{\circ}\right]$. Klotz and Jiang (2017) also found that the asymmetry resulting from shear tends to be downshear left or upshear left, but they used SHIPS data rather than GFS data.

The final key observation is that the wavenumber-1 asymmetry resulting from shear is smaller in the $V_{\text {tr }}$ $(\mathrm{TV}+\mathrm{WVN}-1)+V_{\mathrm{sh}}(\mathrm{WVN}-1)$ model than in the $V_{\text {tr }}(\mathrm{TV})+V_{\text {sh }}(\mathrm{WVN}-1)$ model. In particular, in the $V_{\text {tr }}(\mathrm{TV}+\mathrm{WVN}-1)+V_{\mathrm{sh}}(\mathrm{WVN}-1)$ model, the intercept term $\hat{x}_{\mathrm{sh}, A}^{(0)}$ is not statistically significant, and the slope term $\hat{x}_{\mathrm{sh}, A}^{(1)}<0.2$. This result indicates that when both the wavenumber-1 component and translation vector are used to model asymmetry resulting from translation, the resultant wavenumber- 1 asymmetry resulting from shear becomes smaller.

\section{c. Model performance and selection}

Now, we compare the performance of the wind field models listed in Table 1. The first performance metric discussed is the CVE of the models (see Table 4). The models ordered from lowest to highest CVE are $V_{\text {tr }}(\mathrm{TV}+\mathrm{WVN}-1)+V_{\mathrm{sh}}(\mathrm{WVN}-1), V_{\mathrm{tr}}(\mathrm{TV}+\mathrm{WVN}-1)$, $V_{\text {tr }}(\mathrm{TV})+V_{\text {sh }}(\mathrm{WVN}-1), V_{\text {tr }}(\mathrm{TV}), V_{\text {tr }}(\mathrm{WVN}-1)$, and mean field. Thus, the model with the lowest CVE is the $V_{\text {tr }}(\mathrm{TV}+\mathrm{WVN}-1)+V_{\mathrm{sh}}(\mathrm{WVN}-1)$ model-asymmetry modeled as the translation vector and wavenumber-1 asymmetries resulting from both translation and shear.

Three key observations are evident from the crossvalidation results. First, addition of wavenumber-1 asymmetries to the wind field model predictably lowers the cross-validation error by decreasing the variance of the residuals. Second, the benchmark $V_{\text {tr }}(\mathrm{TV})$ model has a lower cross-validation error than the $V_{\mathrm{tr}}(\mathrm{WVN}-1)$ model. The main reason why the $V_{\text {tr }}(\mathrm{TV})$ model is better-performing is that its mean residual is 0.9 lower than that of the mean field (indicating a smaller model bias). Third, for the GFS data, the difference in crossvalidation error is small when comparing the $V_{\mathrm{tr}}(\mathrm{TV})+$ $V_{\text {sh }}(\mathrm{WVN}-1)$ model to the $V_{\mathrm{tr}}(\mathrm{TV})$ model, or the $V_{\mathrm{tr}}$ $(\mathrm{TV}+\mathrm{WVN}-1)+V_{\mathrm{sh}}(\mathrm{WVN}-1)$ model to the $V_{\mathrm{tr}}(\mathrm{TV}+$ WVN-1) model. Thus, adding shear-induced asymmetry only marginally decreases the cross-validation error when using the GFS data. 

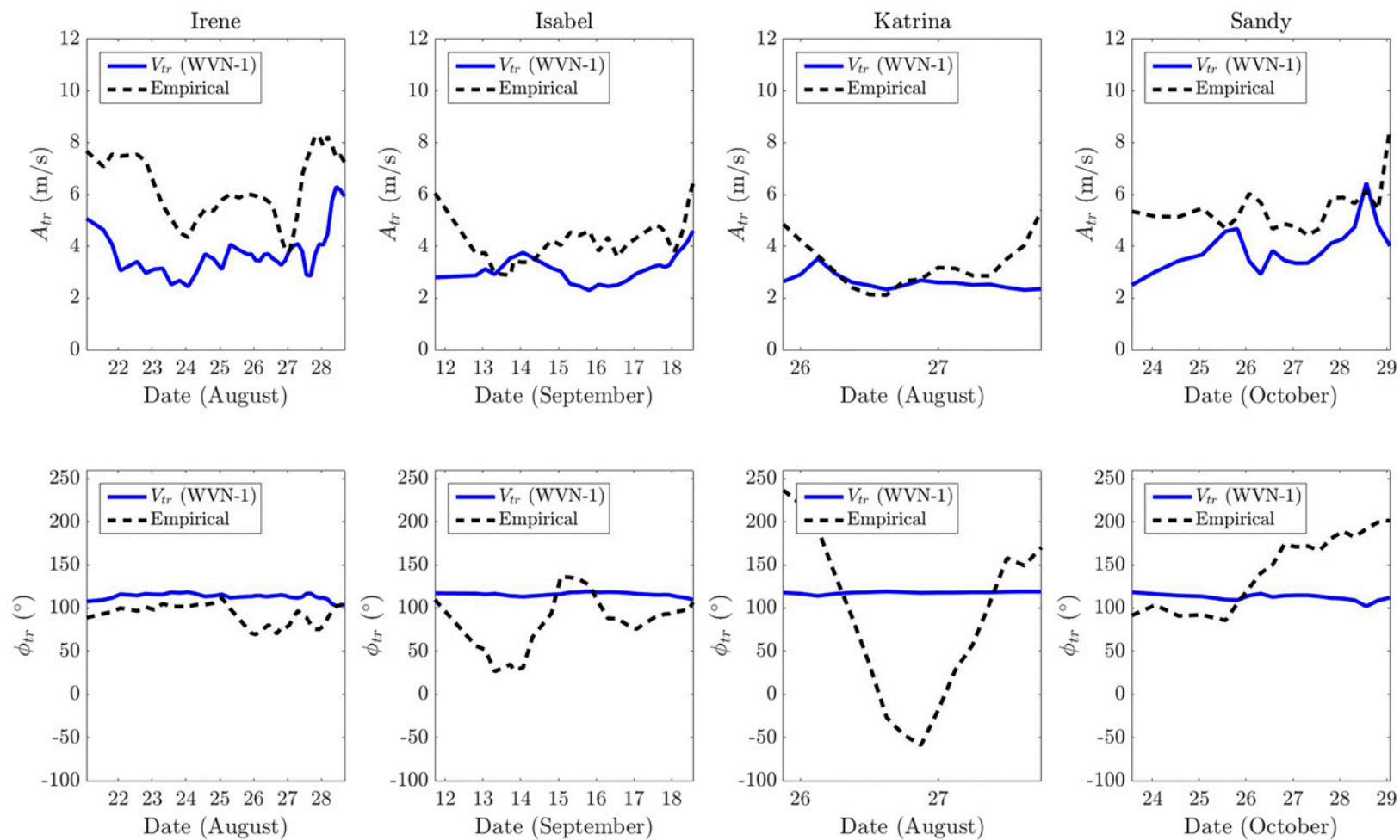

FIG. 5. Wavenumber-1 amplitudes and phases resulting from translation ( $A_{\mathrm{tr}}$ and $\phi_{\mathrm{tr}}$, respectively) for HWind testing storms, as estimated by the $V_{\mathrm{tr}}(\mathrm{WVN}-1)$ model. Empirically estimated amplitudes and phases at the radius of maximum winds are also plotted for comparison purposes.

The second metric discussed is the BIC. A lower BIC indicates a better model. The $V_{\mathrm{tr}}(\mathrm{TV}+\mathrm{WVN}-1)+$ $V_{\text {sh }}(\mathrm{WVN}-1)$ model has the lowest BIC, followed by the $V_{\text {tr }}(\mathrm{TV}+\mathrm{WVN}-1)$ model. Wind field models with more parameters have lower BIC values: as the number of parameters increases, the penalty term for overfitting in the BIC grows at a slower rate compared to the decrease in the objective (see appendix B). The penalty term grows relatively slowly because the number of velocity data points $N$ is much larger than the number of parameters $M$ in our model. ${ }^{5}$

Next, we discuss the performance of the models on the HWind test data as measured by mean-squared error (see Table 4). The models with the lowest MSE under both GFS and SHIPS are $V_{\mathrm{tr}}(\mathrm{TV})+V_{\mathrm{sh}}(\mathrm{WVN}-1)$ for Irene, $V_{\mathrm{tr}}(\mathrm{TV}+\mathrm{WVN}-1)+V_{\mathrm{sh}}(\mathrm{WVN}-1)$ for Isabel and Sandy, and the mean field for Katrina. Thus, the bestperforming models on all storms except Katrina include asymmetry resulting from both translation and shear.

\footnotetext{
${ }^{5}$ As a result of spatial smoothing, the wind field estimation error among physically neighboring locations may be correlated, which results in the "effective" number of data points being smaller than $N$.
}

Specifically, the $V_{\text {tr }}(\mathrm{TV}+\mathrm{WVN}-1)+V_{\text {sh }}(\mathrm{WVN}-1)$ model has the lowest mean-squared error averaged over the four HWind test storms. Figures 3 and 4 demonstrate how the mean-squared error varies over time for each storm.

Figures 5-8 compare the CNLS- and empirically estimated amplitudes and phases at the radius of maximum winds. The figures demonstrate that the mean field is the best for Katrina, because the estimated relationships between the phases and environmental inputs did not accurately estimate the phases of the Katrina snapshots. In addition, the CNLS-estimated amplitudes tend to be smaller than the empirical amplitudes. This is because wavenumber- 1 asymmetries at the radius of maximum winds and other radii can differ; thus CNLS-estimated amplitudes are smaller in order to decrease inaccuracies in velocity estimates introduced by radial variations in asymmetry.

Last, we discuss the MSE of the models on the WRF test data. Because the wind field models are trained using HWind data, test performance on WRF storms is worse than on HWind storms. Models estimated using HWind velocity data are not able to capture the greater azimuthal variability in wind velocities present in the 

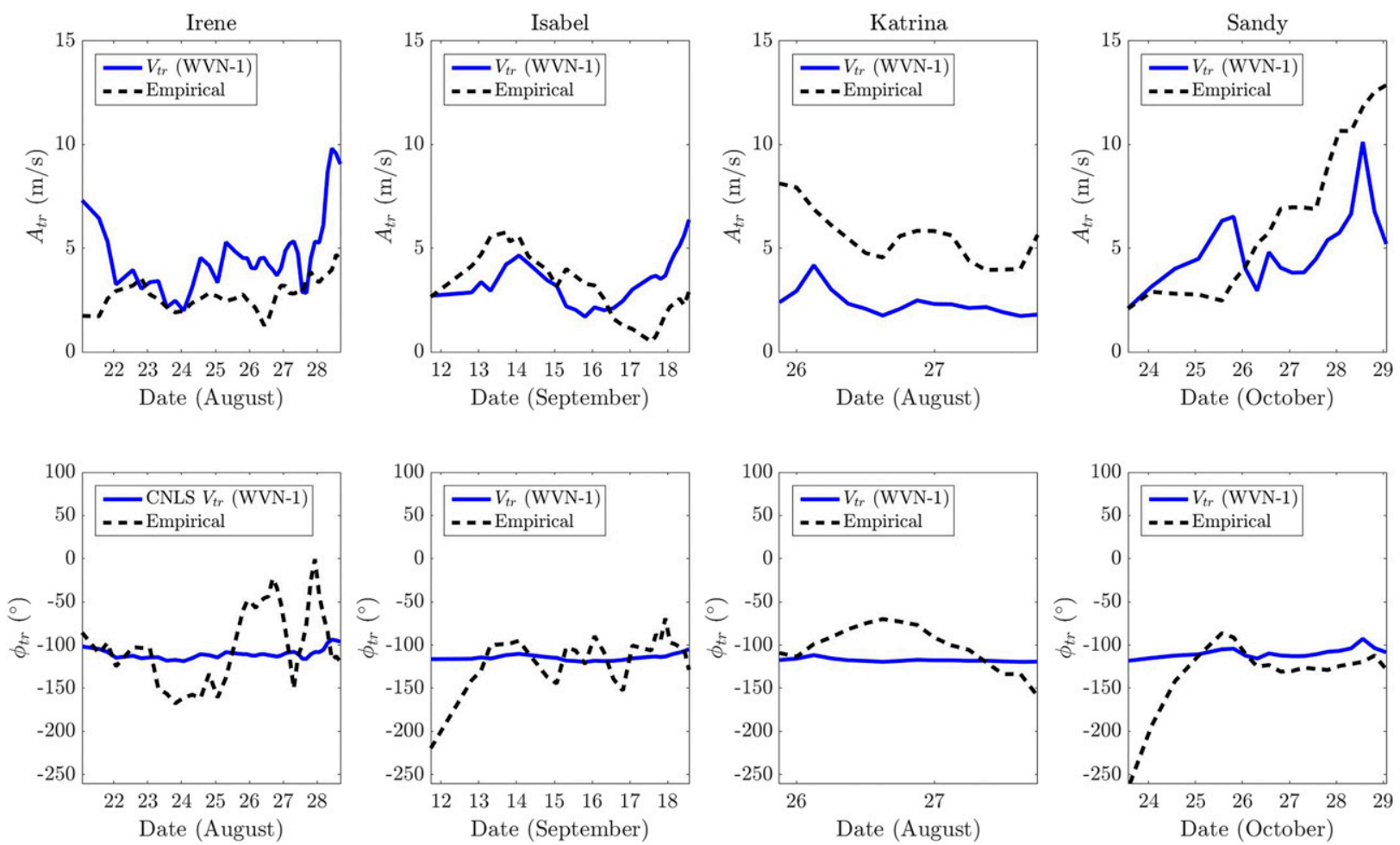

FIG. 6. As in Fig. 5, but as estimated by the $V_{\text {tr }}(\mathrm{TV}+\mathrm{WVN}-1)$ model.

WRF data. Converting the WRF data to 1-min sustained winds, as was discussed in section $2 \mathrm{~d}$, did not noticeably change the velocities. The azimuthal variability is reflected in the empirical asymmetric amplitudes $A_{\text {tr }}$ and $A_{\text {sh }}$ estimated at the radius of maximum winds. The HWind amplitudes for Katrina (and Sandy, in parentheses) using GFS data are $A_{\mathrm{tr}}=3.13$ (4.20) and $A_{\mathrm{sh}}=$ 2.65 (2.12). In contrast, the WRF amplitudes for Katrina and Sandy using GFS data are $A_{\text {tr }}=7.08$ (5.10) and $A_{\text {sh }}=4.44$ (10.73).

The results suggest that the best model is the $V_{\text {tr }}$ $(\mathrm{TV}+\mathrm{WVN}-1)+V_{\text {sh }}(\mathrm{WVN}-1)$ model. However, the CVEs of the models including wavenumber-1 resulting from shear are only marginally better than those of models that do not include shear. For this reason, we perform one-way analysis of variance (ANOVA) as a measure of whether the differences in performance between two models are significant. ${ }^{6}$ According to the oneway ANOVA, there is not a significant difference in performance between the $V_{\mathrm{tr}}(\mathrm{TV}+\mathrm{WVN}-1)+V_{\mathrm{sh}}$ $(\mathrm{WVN}-1)$ and $V_{\mathrm{tr}}(\mathrm{TV}+\mathrm{WVN}-1)$ models, which is evident in the very similar wind fields estimated by these

\footnotetext{
${ }^{6}$ Here, the one-way ANOVA tests whether there is a statistically significant difference between the mean residuals of two different models.
}

two models in Fig. 9. Similarly, there is not a significant performance difference between the $V_{\mathrm{tr}}(\mathrm{TV})+$ $V_{\text {sh }}(\mathrm{WVN}-1)$ and $V_{\text {tr }}(\mathrm{TV})$ models. Parameter estimation using a larger training set and/or evaluating the parameters on more test storms would permit a more definitive conclusion on the effect of shear on asymmetry.

\section{Application of model to wind field simulation}

Given an ensemble consisting of a large number (typically $>10^{3}$ ) of synthetic storm tracks, the asymmetric surface wind field can be simulated at points along each track using the model proposed in this paper. Storm tracks can be generated using the method proposed in Emanuel et al. (2006). After gradient wind fields are simulated along the tracks using our model, a suitable boundary layer model such as the model proposed by Vickery et al. (2009) may be used to convert the gradient winds to surface winds.

To simulate the mean field winds along each track would require estimation of the Holland parameters. The parameters $\bar{V}_{m}$ and $R_{m}$ can be estimated using coupled intensity models (Emanuel et al. 2004; Emanuel 2017). The parameter $B$ can be estimated as a function of the central pressure difference and $V_{m}$, as suggested by Holland et al. (2010). The parameter $S(r)$ can be 

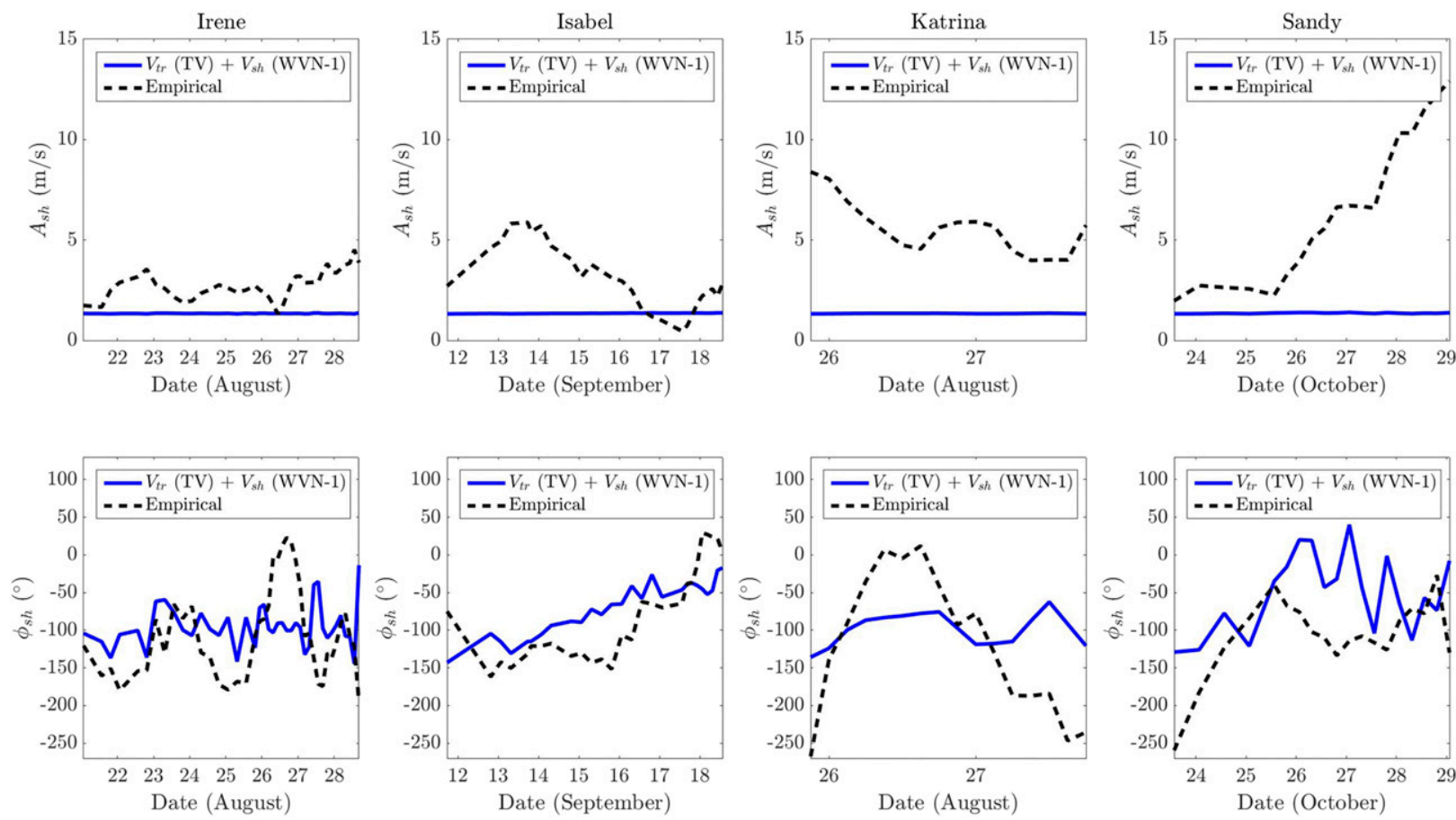

FIG. 7. Wavenumber-1 amplitudes and phases resulting from shear ( $A_{\mathrm{sh}}$ and $\phi_{\mathrm{sh}}$, respectively) for HWind testing storms and GFS data, as estimated by the $V_{\mathrm{tr}}(\mathrm{TV})+V_{\mathrm{sh}}(\mathrm{WVN}-1)$ model. Empirically estimated amplitudes and phases at the radius of maximum winds are also plotted for comparison purposes.

estimated as a function of $r_{0}$, the outer radius at which wind velocity is zero. Finally, a lognormal distribution is recommended for drawing the radius $r_{0}$ (Emanuel 1989; Dean et al. 2009).

To simulate the asymmetries in the wind field along the track, one can use the estimated $V_{\text {tr }}(\mathrm{TV}+\mathrm{WVN}-1)+V_{\text {sh }}$ (WVN-1) model in Table $3 .^{7}$ Then, the asymmetries can be simulated provided that the inputs from storm translation and wind shear are available. To further improve estimation of asymmetries, one can set the amplitudes and phases to be a function of the asymmetries at a previous time step, as well as the shear and translation at the current time. Our model can be suitably extended to account for the dependence of asymmetry on the wind field at previous times.

\section{Conclusions and future work}

In this article, we proposed an asymmetric wind field model for the purpose of wind field estimation along

\footnotetext{
${ }^{7}$ This is the best-performing model, as discussed in section 5c. We elect to use the model that was estimated using GFS because the wind shear obtained using GFS analyses with vortex inversion is a more accurate estimate than using SHIPS.
}

hurricane tracks. Our model incorporates wavenumber-1 asymmetries as a function of storm-translation speed and wind shear, modulating maximum intensity $V_{m}$ in the Holland equation. To obtain parameter estimates for our model, we formulate a constrained, nonlinear least squares optimization problem in which the objective function is the sum of squared errors between model-estimated and observationally based velocity values.

The main contribution of our work is a systematic approach to asymmetric hurricane wind field estimation for the purpose of simulating the wind field along statistically generated tracks in an ensemble. We fit a parametric model to past hurricane velocity wind fields and use environmental variables to estimate asymmetry. Specifically, our asymmetry model captures the wavenumber-1 asymmetric structure with or without inclusion of the translation vector in the model. Below we summarize our key findings:

- Inclusion of the translation vector in the wind field affects the wavenumber- 1 asymmetry resulting from translation. If the translation vector is not added to the winds, the wavenumber- 1 phase occurs slightly more than $90^{\circ}$ clockwise of the translation direction and rotates downwind with increasing translation speed. If the translation vector is added, the wavenumber-1 

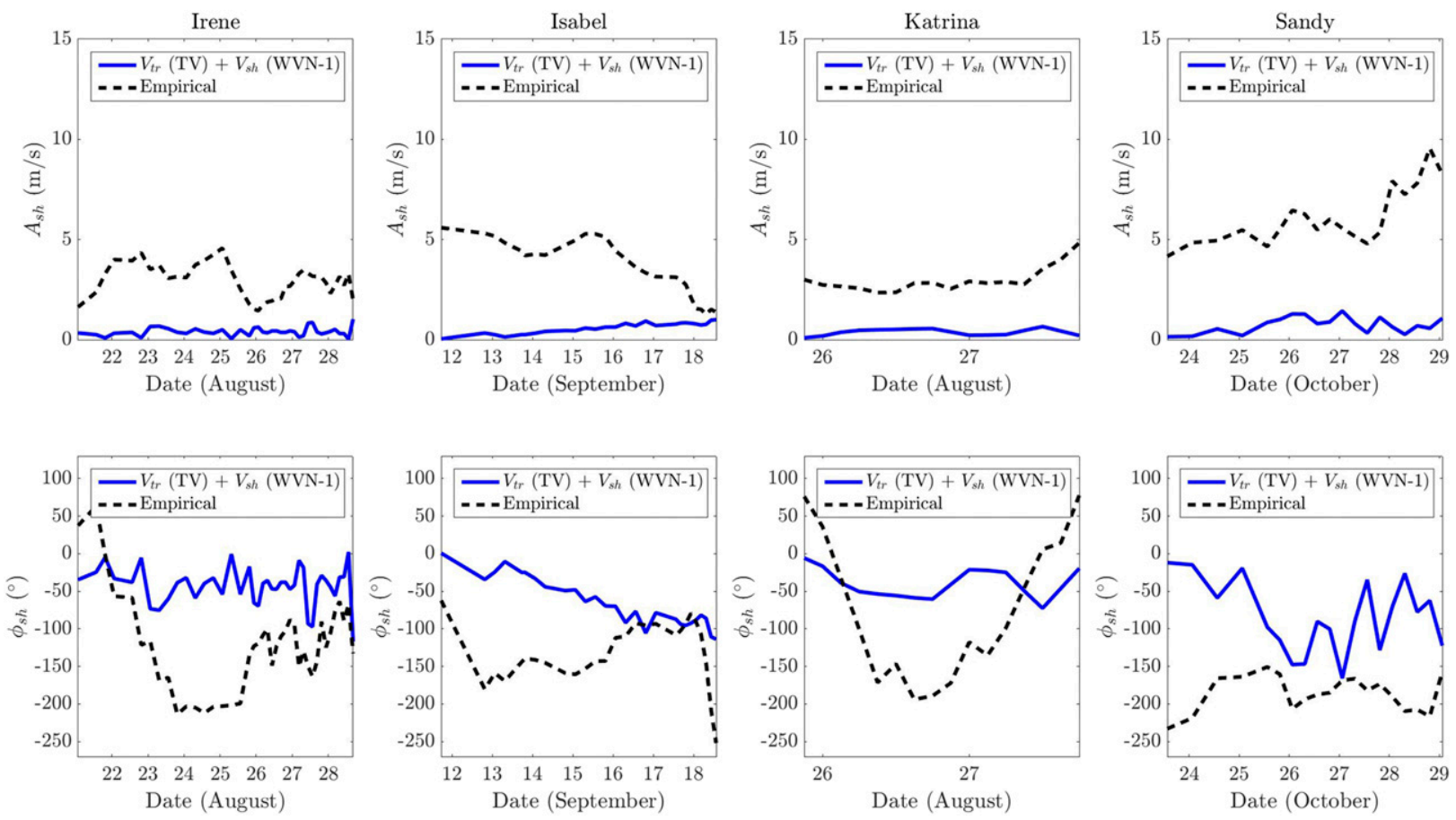

FIG. 8. As in Fig. 7, but as estimated by the $V_{\mathrm{tr}}(\mathrm{TV}+\mathrm{WVN}-1)+V_{\mathrm{sh}}(\mathrm{WVN}-1)$ model

component estimates the residual asymmetry that remains in the wind field. The phase of this residual asymmetry occurs slightly more than $90^{\circ}$ counterclockwise of the translation direction and rotates upwind with increasing translation speed.

- When using SHIPS data, the wavenumber-1 shearrelative phase rotates downwind from downshear to left of shear with increasing shear magnitude. When using GFS data, the phase rotates from downshear to left of shear with increasing shear magnitude if the wavenumber-1 component resulting from translation is included, but it rotates upwind from upshear left to downshear left with increasing shear magnitude otherwise. The asymmetry resulting from shear is substantially smaller when the wavenumber- 1 asymmetry resulting from translation is included.

- To determine the best model, we evaluate models using the cross-validation error, Bayesian information criterion, and mean-squared error on test storms as metrics. The procedure suggests that the bestperforming model is the $V_{\text {tr }}(\mathrm{TV}+\mathrm{WVN}-1)+V_{\text {sh }}$ (WVN-1) model, which accounts for the translation vector as well as wavenumber-1 asymmetries resulting from translation and shear. However, omission of the wavenumber-1 component resulting from shear from this model only marginally decreases the model performance. Additional data for estimation would permit us to generate more conclusive results on asymmetry resulting from shear.

- Model performance is relatively weak on WRF data, because all the models are trained using HWind data. Since WRF velocity data are instantaneous winds, WRF snapshots have greater azimuthal variability that is not captured by models estimated using HWind data. Additional WRF storms would allow us to draw more decisive conclusions on the effect of using HWind versus WRF snapshots in parameter estimation.

Future work on modeling asymmetry should account for the dependence of the amplitudes and phases on previous time steps, as discussed in section 6 . Furthermore, stochastic methods can be introduced for modeling the radial variability in the translation and shear-induced phase with increasing radius. In particular, shear is likely to have a substantial effect on storm asymmetry outside of the radius of maximum winds (Klotz and Jiang 2017). Also, modeling secondary eyewalls in storms might bring additional improvements to wind field estimation as compared to asymmetry. By improving asymmetry modeling techniques and/or incorporating additional physical features into the wind field model, we hope to further increase the accuracy of hurricane wind field estimation for engineering risk assessment. 

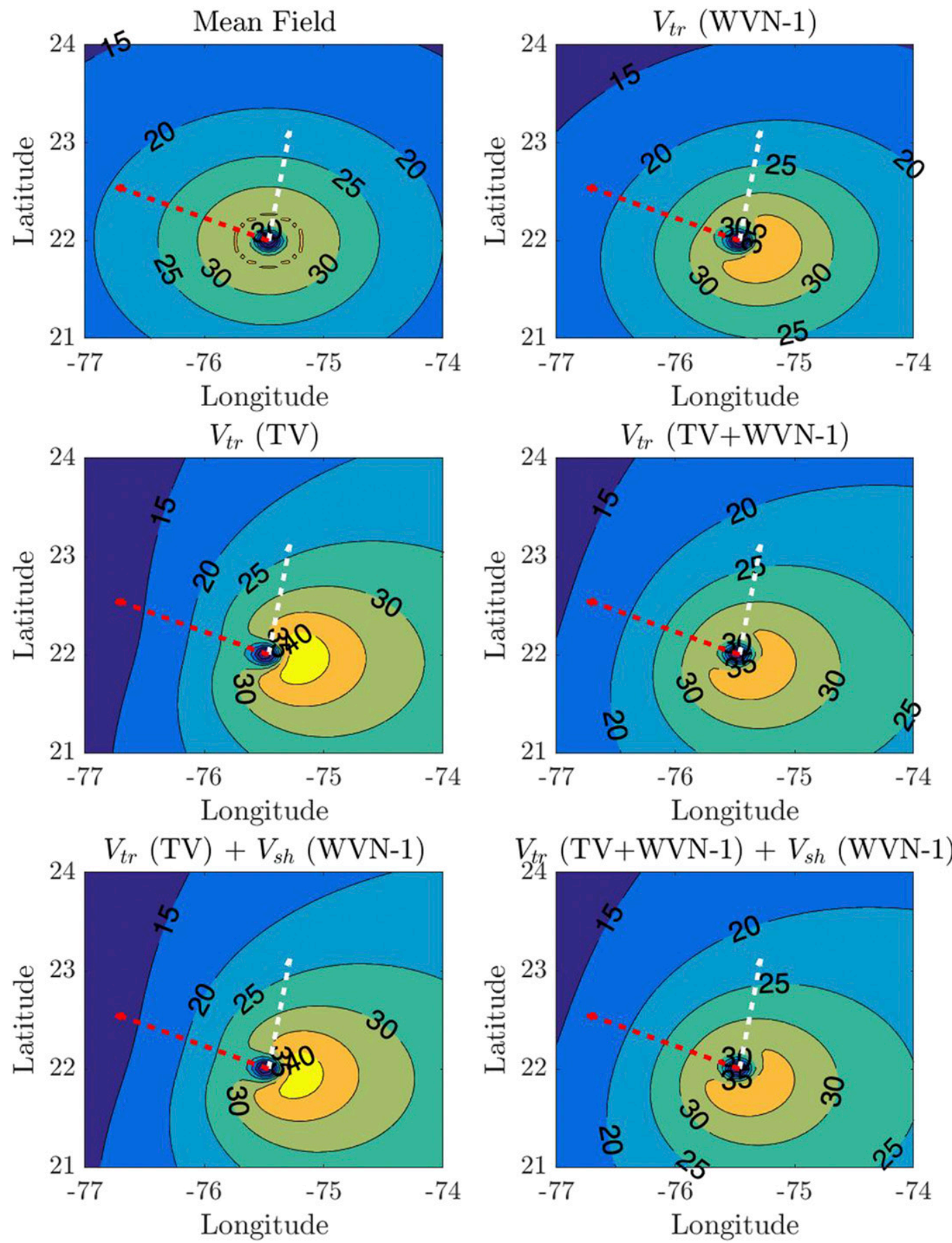

FIG. 9. Visualization of 2D gradient wind field estimation. The plots show the estimated wind field of Hurricane Sandy at 163025 Oct using the following models: (top left) mean field, (top right) $V_{\operatorname{tr}}(\mathrm{WVN}-1$ ) model, (middle left) $V_{\mathrm{tr}}(\mathrm{TV})$ model, (middle right) $V_{\mathrm{tr}}\left(\mathrm{TV}+\mathrm{WVN}-1\right.$ ) model, (bottom left) $V_{\mathrm{tr}}(\mathrm{TV})+$ $V_{\text {sh }}(\mathrm{WVN}-1)$ model, and (bottom right) $V_{\text {tr }}(\mathrm{TV}+\mathrm{WVN}-1)+V_{\text {sh }}(\mathrm{WVN}-1)$ model. The plots show the translation vector (white) and shear vector (red), with the length of the vectors indicating their relative magnitudes. 
Acknowledgments. This material is based upon work supported by NSF FORCES Award CNS-1239054, NSF CAREER Award CNS 1453126, and the NSF Graduate Research Fellowship under Grant 1122374. The third author was supported by NSF Grant AGS-1418508. Any opinion, findings, and conclusions or recommendations expressed in this material are those of the authors and do not necessarily reflect the views of the National Science Foundation. The authors thank Professor Fuqing Zhang at The Pennsylvania State University for providing the Katrina and Sandy WRF datasets, and Jonathan Lin in the Department of Earth, Atmospheric, and Planetary Sciences at the Massachusetts Institute of Technology for providing the wind shear datasets. In addition, the authors thank Mark Powell for his work maintaining and developing the HWind database. Also, we thank four anonymous reviewers for helping us to improve this work.

\section{APPENDIX A}

\section{Objective Function-Jacobian and Hessian}

Recall in section 3 that to minimize the CNLS objective $f(\mathbf{x})$, we supply the gradient $\nabla f(\mathbf{x})$ and Hessian $\nabla^{2} f(\mathbf{x})$ to the solver [see Eq. (11)]. These derivatives require the residual vector $e(\mathbf{x})$, Jacobian $J(\mathbf{x})$, and second derivative of the residuals $\nabla^{2} e(\mathbf{x})$. The residual vector $e(\mathbf{x})$ was defined for the wind field model in section 3 . The Jacobian for velocity data point $i, \nabla e_{i}(\mathbf{x})$, is one row of $J(\mathbf{x})$ and is given as $\left[J_{i}\left(\mathbf{x}_{\mathrm{tr}}\right)^{\mathrm{T}}, J_{i}\left(\mathbf{x}_{\mathrm{sh}}\right)^{\mathrm{T}}\right]^{\mathrm{T}}$, where $J_{i}\left(\mathbf{x}_{\mathrm{tr}}\right)=\left[J_{i}\left(\mathbf{x}_{\mathrm{tr}, A}\right)^{\mathrm{T}}, J_{i}\left(\mathbf{x}_{\mathrm{tr}, \phi}\right)^{\mathrm{T}}\right]^{\mathrm{T}}$ and $J_{i}\left(\mathbf{x}_{\mathrm{sh}}\right)=$ $\left[J_{i}\left(\mathbf{x}_{\mathrm{sh}, A}\right)^{\mathrm{T}}, J_{i}\left(\mathbf{x}_{\mathrm{sh}, \phi}\right)^{\mathrm{T}}\right]^{\mathrm{T}}$. Furthermore,

$$
\begin{aligned}
J_{i}\left(\mathbf{x}_{\mathrm{tr}, A}\right)= & -Y_{i} \cos \left\{\lambda_{\mathrm{tr}, i}-\phi_{\mathrm{tr}}\left[V_{\mathrm{tr}, t(i)} ; x_{\mathrm{tr}, \phi}\right]\right\} d_{\mathrm{tr}, t(i)} \\
J_{i}\left(\mathbf{x}_{\mathrm{tr}, \phi}\right)= & -Y_{i} A_{\mathrm{tr}}\left[V_{\mathrm{tr}, t(i)} ; \mathbf{x}_{\mathrm{tr}, A}\right] \sin \left\{\lambda_{\mathrm{tr}, i}\right. \\
& \left.-\phi_{\mathrm{tr}}\left[V_{\mathrm{tr}, t(i)} ; \mathbf{x}_{\mathrm{tr}, \phi}\right]\right\} d_{\mathrm{tr}, t(i)} \\
J_{i}\left(\mathbf{x}_{\mathrm{sh}, A}\right)= & -Y_{i} \cos \left\{\lambda_{\mathrm{sh}, i}-\phi_{\mathrm{sh}}\left[V_{\mathrm{sh}, t(i)} ; \mathbf{x}_{\mathrm{sh}, \phi}\right]\right\} d_{\mathrm{sh}, t(i)} \\
J_{i}\left(\mathbf{x}_{\mathrm{sh}, \phi}\right)= & -Y_{i} A_{\mathrm{sh}}\left[V_{\mathrm{sh}, t(i)} ; \mathbf{x}_{\mathrm{sh}, A}\right] \sin \left\{\lambda_{\mathrm{sh}, i}\right. \\
& \left.-\phi_{\mathrm{sh}}\left[V_{\mathrm{sh}, t(i)} ; \mathbf{x}_{\mathrm{sh}, \phi}\right]\right\} d_{\mathrm{sh}, t(i)},
\end{aligned}
$$

where $d_{\mathrm{tr}, t(i)}=\left[1, V_{\mathrm{tr}, t(i)}\right]^{\mathrm{T}}, d_{\mathrm{sh}, t(i)}=\left[1, V_{\mathrm{sh}, t(i)}\right]^{\mathrm{T}}$, and $Y_{i}$ is used to denote $Y_{i}\left[r_{i}, \mathbf{s}_{i}\left(r_{i}\right)\right]$, or the mean field component for data point $i$. The second derivatives of the residuals are given as

$$
\nabla^{2} e_{i}(\mathbf{x})=\left[\begin{array}{cc}
\frac{\partial^{2} e_{i}(\mathbf{x})}{\partial \mathbf{x}_{\mathrm{tr}}^{2}} & \mathbf{0} \\
\mathbf{0} & \frac{\partial^{2} e_{i}(\mathbf{x})}{\partial \mathbf{x}_{\mathrm{sh}}^{2}}
\end{array}\right],
$$

where

$$
\begin{gathered}
\frac{\partial^{2} e_{i}(\mathbf{x})}{\partial \mathbf{x}_{\mathrm{tr}}^{2}}=\left\{\begin{array}{cc}
\mathbf{0} & \frac{\partial^{2} e_{i}(\mathbf{x})}{\partial \mathbf{x}_{\mathrm{tr}, A} \partial \mathbf{x}_{\mathrm{tr}, \phi}} \\
{\left[\frac{\partial^{2} e_{i}(\mathbf{x})}{\partial \mathbf{x}_{\mathrm{tr}, A} \partial \mathbf{x}_{\mathrm{tr}, \phi}}\right]^{\mathrm{T}}} & \frac{\partial^{2} e_{i}(\mathbf{x})}{\partial \mathbf{x}_{\mathrm{tr}, \phi}^{2}}
\end{array}\right\}, \\
\frac{\partial^{2} e_{i}(\mathbf{x})}{\partial \mathbf{x}_{\mathrm{sh}}^{2}}=\left\{\begin{array}{cc}
\mathbf{0} & \frac{\partial^{2} e_{i}(\mathbf{x})}{\partial \mathbf{x}_{\mathrm{sh}, A} \partial \mathbf{x}_{\mathrm{sh}, \phi}} \\
{\left[\frac{\partial^{2} e_{i}(\mathbf{x})}{\partial \mathbf{x}_{\mathrm{sh}, A} \partial \mathbf{x}_{\mathrm{sh}, \phi}}\right]^{\mathrm{T}}} & \frac{\partial^{2} e_{i}(\mathbf{x})}{\partial \mathbf{x}_{\mathrm{sh}, \phi}^{2}}
\end{array}\right\} .
\end{gathered}
$$

Furthermore,

$$
\begin{aligned}
\frac{\partial^{2} e_{i}(\mathbf{x})}{\partial \mathbf{x}_{\mathrm{tr}, A} \partial \mathbf{x}_{\mathrm{tr}, \phi}}= & -Y_{i} \sin \left\{\lambda_{\mathrm{tr}, i}\right. \\
& \left.-\phi_{\mathrm{tr}}\left[V_{\mathrm{tr}, t(i)} ; \mathbf{x}_{\mathrm{tr}, \phi}\right]\right\}\left[d_{\mathrm{tr}, t(i)}\right]^{\mathrm{T}}\left[d_{\mathrm{tr}, t(i)}\right] \\
\frac{\partial^{2} e_{i}(\mathbf{x})}{\partial \mathbf{x}_{\mathrm{tr}, \phi}^{2}}= & Y_{i} A_{\mathrm{tr}}\left[V_{\mathrm{tr}, t(i)} ; \mathbf{x}_{\mathrm{tr}, A}\right] \cos \left\{\lambda_{\mathrm{tr}, i}\right. \\
& \left.-\phi_{\mathrm{tr}}\left[V_{\mathrm{tr}, t(i)} ; \mathbf{x}_{\mathrm{tr}, \phi}\right]\right\}\left[d_{\mathrm{tr}, t(i)}\right]^{\mathrm{T}}\left[d_{\mathrm{tr}, t(i)}\right] \\
\frac{\partial^{2} e_{i}(\mathbf{x})}{\partial \mathbf{x}_{\mathrm{sh}, A} \partial \mathbf{x}_{\mathrm{sh}, \phi}=} & -Y_{i} \sin \left\{\lambda_{\mathrm{sh}, i}\right. \\
& \left.-\phi_{\mathrm{sh}}\left[V_{\mathrm{sh}, t(i)} ; \mathbf{x}_{\mathrm{sh}, \phi}\right]\right\}\left[d_{\mathrm{sh}, t(i)}\right]^{\mathrm{T}}\left[d_{\mathrm{sh}, t(i)}\right] \\
\frac{\partial^{2} e_{i}(\mathbf{x})}{\partial \mathbf{x}_{\mathrm{sh}, \phi}^{2}}= & Y_{i} A_{\mathrm{sh}}\left[V_{\mathrm{sh}, t(i)} ; \mathbf{x}_{\mathrm{sh}, A}\right] \cos \left\{\lambda_{\mathrm{sh}, i}\right. \\
& \left.-\phi_{\mathrm{sh}}\left[V_{\mathrm{sh}, t(i)} ; \mathbf{x}_{\mathrm{sh}, \phi}\right]\right\}\left[d_{\mathrm{sh}, t(i)}\right]^{\mathrm{T}}\left[d_{\mathrm{sh}, t(i)}\right] . \\
& \operatorname{APPENDIX~B}
\end{aligned}
$$

\section{APPENDIX B}

\section{Bayesian Information Criterion}

The BIC is defined as

$$
\mathrm{BIC}=-2 \ln (\hat{L})+\ln (N) M,
$$

where $\hat{L}$ is the maximum likelihood of the model, $N$ is the number of data points, and $M$ is the number of model parameters. A lower value of the BIC corresponds to a higher-quality model.

The sum of squared residuals is the metric minimized using the CNLS method [Eq. (11)], but computing the BIC requires the model's maximum likelihood. Thus we 
estimate $\ln (\hat{L})$ to be the maximum log-likelihood of a Gaussian model, for which the estimation error at each data point $i$ is independently distributed with mean $e_{i}(\mathbf{x})$ and variance $\hat{\sigma}^{2}$ :

$$
\ln (\hat{L})=-\frac{N}{2} \ln \left(2 \pi \hat{\sigma}^{2}\right)-\frac{1}{2 \hat{\sigma}^{2}} \sum_{i=1}^{N} e_{i}^{2}(\hat{\mathbf{x}}) .
$$

The parameter $\hat{\sigma}$ is the empirical standard deviation of the residuals. The BIC is computed for each model, and the minimum $\mathrm{BIC}$ is denoted as $\overline{\mathrm{BIC}}$. Then, the final metric reported is the probability of each model $m$ divided by the probability of the best-performing model, given by $\exp \left[-0.5\left(\mathrm{BIC}_{m}-\overline{\mathrm{BIC}}\right)\right]$. See Burnham and Anderson (2004) for further details.

\section{REFERENCES}

Alvehag, K., and L. Soder, 2011: A reliability model for distribution systems incorporating seasonal variations in severe weather. IEEE Trans. Power Delivery, 26, 910-919, https:// doi.org/10.1109/TPWRD.2010.2090363.

Bender, M. A., T. R. Knutson, R. E. Tuleya, J. J. Sirutis, G. A. Vecchi, S. T. Garner, and I. M. Held, 2010: Modeled impact of anthropogenic warming on the frequency of intense Atlantic hurricanes. Science, 327, 454-458, https://doi.org/ 10.1126/science. 1180568 .

Burnham, K. P., and D. R. Anderson, 2004: Multimodel inference: Understanding AIC and BIC in model selection. Sociol. Methods Res., 33, 261-304, https://doi.org/10.1177/ 0049124104268644

Campbell, R. J., and S. Lowry, 2012: Weather-related power outages and electric system resiliency. Congressional Research Service Rep., 15 pp., https://fas.org/sgp/crs/misc/ R42696.pdf.

Casson, E., and S. Coles, 2000: Simulation and extremal analysis of hurricane events. J. Roy. Stat. Soc., 49C, 227-245, https:// doi.org/10.1111/1467-9876.00189.

Chavas, D. R., N. Lin, and K. Emanuel, 2015: A model for the complete radial structure of the tropical cyclone wind field. Part I: Comparison with observed structure. J. Atmos. Sci., 72, 3647-3662, https://doi.org/10.1175/JAS-D-15-0014.1.

Dean, L., K. A. Emanuel, and D. R. Chavas, 2009: On the size distribution of Atlantic tropical cyclones. Geophys. Res. Lett., 36, L14803, https://doi.org/10.1029/2009GL039051.

DeMaria, M., M. Mainelli, L. K. Shay, J. A. Knaff, and J. Kaplan, 2005: Further improvements to the Statistical Hurricane Intensity Prediction Scheme (SHIPS). Wea. Forecasting, 20, 531-543, https://doi.org/10.1175/WAF862.1.

DiNapoli, S. M., M. A. Bourassa, and M. D. Powell, 2012: Uncertainty and intercalibration analysis of $\mathrm{H}^{*}$ Wind. J. Atmos. Oceanic Technol., 29, 822-833, https://doi.org/10.1175/JTECHD-11-00165.1.

Emanuel, K. A., 1989: The finite-amplitude nature of tropical cyclogenesis. J. Atmos. Sci., 46, 3431-3456, https://doi.org/ 10.1175/1520-0469(1989)046<3431:TFANOT>2.0.CO;2.

, 2004: Tropical cyclone energetics and structure. Atmospheric Turbulence and Mesoscale Meteorology, E. Fedorovich, R. Rotunno, and B. Stevens, Eds., Cambridge University Press, 165-192, https://doi.org/10.1017/CBO9780511735035.010.
_ 2017: A fast intensity simulator for tropical cyclone risk analysis. Nat. Hazards, 88, 779-796, https://doi.org/10.1007/ s11069-017-2890-7.

, C. Desautels, C. Holloway, and R. Korty, 2004: Environmental control of tropical cyclone intensity. J. Atmos. Sci., 61, 843-858, https://doi.org/10.1175/1520-0469(2004)061<0843:ECOTCI > 2.0.CO;2.

, S. Ravela, E. Vivant, and C. Risi, 2006: A statistical deterministic approach to hurricane risk assessment. Bull. Amer. Meteor. Soc., 87, 299-314, https://doi.org/10.1175/BAMS-87-3-299.

Galarneau, T. J., and C. A. Davis, 2013: Diagnosing forecast errors in tropical cyclone motion. Mon. Wea. Rev., 141, 405-430, https://doi.org/10.1175/MWR-D-12-00071.1.

Green, B. W., and F. Zhang, 2013: Impacts of air-sea flux parameterizations on the intensity and structure of tropical cyclones Mon. Wea. Rev., 141, 2308-2324, https://doi.org/10.1175/MWRD-12-00274.1.

Holland, G. J., 1980: An analytic model of the wind and pressure profiles in hurricanes. Mon. Wea. Rev., 108, 1212-1218, https://doi.org/ 10.1175/1520-0493(1980)108<1212:AAMOTW >2.0.CO;2.

- J. I. Belanger, and A. Fritz, 2010: A revised model for radial profiles of hurricane winds. Mon. Wea. Rev., 138, 4393-4401, https://doi.org/10.1175/2010MWR3317.1.

Klotz, B. W., and H. Jiang, 2017: Examination of surface wind asymmetries in tropical cyclones. Part I: General structure and wind shear impacts. Mon. Wea. Rev., 145, 3989-4009, https:// doi.org/10.1175/MWR-D-17-0019.1

— and D. S. Nolan, 2019: SFMR surface wind undersampling over the tropical cyclone life cycle. Mon. Wea. Rev., 147, 247268, https://doi.org/10.1175/MWR-D-18-0296.1.

Landsea, C. W., and J. L. Franklin, 2013: Atlantic hurricane database uncertainty and presentation of a new database format. Mon. Wea. Rev., 141, 3576-3592, https://doi.org/10.1175/ MWR-D-12-00254.1.

Munsell, E. B., and F. Zhang, 2014: Prediction and uncertainty of Hurricane Sandy (2012) explored through a real-time cloudpermitting ensemble analysis and forecast system assimilating airborne Doppler radar observations. J. Adv. Model. Earth Syst., 6, 38-58, https://doi.org/10.1002/2013MS000297.

Nocedal, J., and S. J. Wright, 2006: Numerical Optimization. Springer, $636 \mathrm{pp}$.

Ouyang, M., 2014: Review on modeling and simulation of interdependent critical infrastructure systems. Reliab. Eng. Syst. Saf., 121, 43-60, https://doi.org/10.1016/j.ress.2013.06.040.

Powell, M. D., S. H. Houston, L. R. Amat, and N. MorisseauLeroy, 1998: The HRD real-time hurricane wind analysis system. J. Wind Eng. Ind. Aerodyn., 77-78, 53-64, https:// doi.org/10.1016/S0167-6105(98)00131-7.

Rousseau-Rizzi, R., and K. Emanuel, 2019: An evaluation of hurricane superintensity in axisymmetric numerical models. J. Atmos. Sci., 76, 1697-1708, https://doi.org/10.1175/JAS-D-18-0238.1.

Schwerdt, R. W., F. P. Ho, and R. R. Watkins, 1979: Meteorological criteria for standard project hurricane and probable maximum hurricane windfields, Gulf and East Coasts of the United States. NOAA Tech. Rep. NWS 23, 317 pp., https:// www.nws.noaa.gov/oh/hdsc/Technical_reports/TR23.pdf.

Shapiro, L. J., 1983: The asymmetric boundary layer flow under a translating hurricane. J. Atmos. Sci., 40, 1984-1998, https:// doi.org/10.1175/1520-0469(1983)040<1984:TABLFU>2.0.CO;2.

Uhlhorn, E. W., B. W. Klotz, T. Vukicevic, P. D. Reasor, and R. F. Rogers, 2014: Observed hurricane wind speed asymmetries and relationships to motion and environmental shear. Mon. Wea. Rev., 142, 1290-1311, https://doi.org/10.1175/MWR-D-13-00249.1. 
Vickery, P. J., P. F. Skerlj, A. C. Steckley, and L. A. Twisdale, 2000a: Hurricane wind field model for use in hurricane simulations. J. Struct. Eng., 126, 1203-1221, https://doi.org/10.1061/ (ASCE)0733-9445(2000)126:10(1203).

_,$\ldots$, and L. A. Twisdale, 2000b: Simulation of hurricane risk in the U.S. using empirical track model. J. Struct. Eng., 126, 1222-1237, https://doi.org/10.1061/(ASCE)0733-9445(2000) 126:10(1222).

- D. Wadhera, M. D. Powell, and Y. Chen, 2009: A hurricane boundary layer and wind field model for use in engineering applications. J. Appl. Meteor. Climatol., 48, 381-405, https:// doi.org/10.1175/2008JAMC1841.1.

Vukicevic, T., E. Uhlhorn, P. Reasor, and B. Klotz, 2014: A novel multiscale intensity metric for evaluation of tropical cyclone intensity forecasts. J. Atmos. Sci., 71, 1292-1304, https:// doi.org/10.1175/JAS-D-13-0153.1.

Watson, C. C., and M. E. Johnson, 2004: Hurricane loss estimation models: Opportunities for improving the state of the art. Bull. Amer. Meteor. Soc., 85, 1713-1726, https://doi.org/10.1175/ BAMS-85-11-1713.

Weng, Y., and F. Zhang, 2012: Assimilating airborne Doppler radar observations with an ensemble Kalman filter for convectionpermitting hurricane initialization and prediction: Katrina
(2005). Mon. Wea. Rev., 140, 841-859, https://doi.org/10.1175/ 2011MWR3602.1.

Willoughby, H. E., and M. E. Rahn, 2004: Parametric representation of the primary hurricane vortex. Part I: Observations and evaluation of the Holland (1980) model. Mon. Wea. Rev., 132, 3033-3048, https://doi.org/10.1175/MWR2831.1.

, R. W. R. Darling, and M. E. Rahn, 2006: Parametric representation of the primary hurricane vortex. Part II: A new family of sectionally continuous profiles. Mon. Wea. Rev., 134, 1102-1120, https://doi.org/10.1175/MWR3106.1.

Xie, L., S. Bao, L. J. Pietrafesa, K. Foley, and M. Fuentes, 2006: A real-time hurricane surface wind forecasting model: Formulation and verification. Mon. Wea. Rev., 134, 13551370, https://doi.org/10.1175/MWR3126.1.

Zhang, P., G. Li, P. B. Luh, W. Li, Z. Bie, and C. Serna, 2014: Risk analysis for distribution systems in the Northeast U.S. under wind storms. 2014 IEEE PES General Meeting-Conf. \& Exposition, National Harbor, MD, IEEE, https://doi.org/ 10.1109/PESGM.2014.6939519.

Zhou, Y., A. Pahwa, and S.-S. Yang, 2006: Modeling weatherrelated failures of overhead distribution lines. IEEE Trans. Power Syst., 21, 1683-1690, https://doi.org/10.1109/ TPWRS.2006.881131. 